\title{
Hopf bifurcations on cubic lattices
}

\author{
T K Callahan \\ Department of Mathematics, University of Michigan, Ann Arbor, MI 48109, USA and \\ Department of Mathematics and Statistics, Arizona State University, Tempe, \\ AZ 85287, $\mathrm{USA}^{1}$ \\ E-mail: timcall@math.la.asu.edu
}

Received 3 March 2003

Published 5 September 2003

Online at stacks.iop.org/Non/16/2099

Recommended by M J Field

\begin{abstract}
Group theoretic means are employed to analyse the Hopf bifurcation on pattern forming systems with the periodicity of the face-centred (FCC) and bodycentred (BCC) cubic lattices. We find all $\mathbb{C}$-axial subgroups of the normal form symmetry group by first extending the symmetry to a larger group. There are 15 such solutions for the FCC lattice, of which at least 12 can be stable for appropriate parameter values. In addition, a number of subaxial solutions can bifurcate directly from the trivial solution, and quasiperiodic solutions can also exist. We find $33 \mathbb{C}$-axial solutions for the BCC lattice and their stability criteria. We discuss applications of the method of symmetry enlargement to other systems. A model-independent approach is taken throughout, and the results are applicable to a wide variety of pattern forming systems. This work is an extension of that done in Callahan T K (2000 Hopf bifurcations on the FCC lattice Proc. Int. Conf. on Differential Equations (Berlin, 1999) vol 1, ed Fiedler et al (Singapore: World Scientific) pp 154-6; 2003 Hopf bifurcations on cubic lattices Bifurcations, Symmetry and Patterns (Trends in Mathematics) ed J Buescu et al (Basel: Birkhauser) pp 123-7).
\end{abstract}

Mathematics Subject Classification: 37G40, 35K57

\section{Introduction}

In recent years pattern formation has been the subject of intense study, and great progress has been made with the use of group theoretic techniques [1]. Equivariant bifurcation theory has

1 Present address. 
been applied to the analysis of systems periodic in two directions, resulting in a relatively complete description of bifurcations on the hexagonal and square lattices [1-3]. These results are model-independent, and apply equally well to Bénard convection, two-dimensional Turing instabilities, the Faraday instability, and many other systems.

Very little corresponding work has been done for the case of patterns periodic in three dimensions, in part because most of the systems (e.g. Bénard convection) have only twodimensional symmetry. This is not true, however, for the Turing instability [4], where chemicals catalyse each other's formation and destruction. If they diffuse at different rates the homogeneous state can lose stability to states with a characteristic length scale: a pattern. This length scale is intrinsic in that it depends only upon the chemical reaction rates and the diffusivities, and not upon the dimensions of the experimental apparatus. Thus the Turing instability can be studied in some fiducial volume far away from the influence of any boundaries, and truly three-dimensional patterns can appear. Indeed, several such patterns have been observed in numerical simulations of the Brusselator model [5,6]. As in the twodimensional case, the simplest solutions that can appear are those that retain the periodicity of some lattice. For the steady-state bifurcation, solutions with the periodicity of the simple (SC), face-centred (FCC) and body-centred (BCC) cubic lattices have been found, and their stabilities analysed [7-10].

It is well-known that in a two-species chemical reaction-diffusion system the static homogeneous state can undergo a Hopf bifurcation only to an oscillating state with zero wavenumber, so that the resulting pattern is still spatially homogeneous. With more than two chemicals (as in the Oregonator model) oscillatory patterns with spatiotemporal symmetries can occur, and it is thus necessary to study the corresponding time-periodic pattern formation problems, as was done for the hexagonal lattice in [11]. In three dimensions the Hopf bifurcation on the SC lattice was analysed in [12]. The forms of the $\mathbb{C}$-axial solutions for the FCC and BCC lattices were found in $[13,14]$ by extending their symmetry groups, and the branching equations for the FCC lattice were determined. In this paper we complete the analysis of these two lattices with detailed descriptions and stability calculations for the various solutions. We also discuss the behaviour of some of the subaxial branches for the FCC latttice. Finally, we explain how the particular group extensions were determined, and how this method can be used to find the $\mathbb{C}$-axial solutions of other systems with high degrees of symmetry.

We start with a chemical reaction-diffusion system that has a spatially homogeneous static state that is stable in some region in parameter space. We assume that as a distinguished parameter is varied modes with the critical wavenumber $k_{\mathrm{c}}$ are the first to lose stability, and that they do so via a Hopf bifurcation. As the other modes are still stable, we would like to perform a centre manifold reduction, slaving the stable modes to the critical ones. Then the dynamics in the centre manifold could be described in terms of the critical modes alone. Unfortunately, the spectrum of allowed wavenumbers is continuous, so the stable eigenvalues are not bounded away from the imaginary axis and the centre manifold theorem cannot be applied. Furthermore, there is a continuous sphere of wavevectors with wavenumber $k_{\mathrm{c}}$. The customary solution is to assume an ansatz of periodicity: we restrict our attention to solutions that have the periodicity of some lattice. This imposition of periodic boundary conditions is justified after the fact by the observation that experiments often produce the solutions that result from this ansatz. Furthermore, the influence of nearby wavevectors can be reintroduced by a study of long wavelength instabilities (eckhaus, zig-zag, skew-varicose), as in [15-17]. The price paid for the ansatz is that we can only determine the stability of a solution on a lattice with respect to perturbations on the same lattice. We cannot, for instance, determine the relative stability of hexagons and squares. Thus, strictly speaking, any stability 
results we find can only guarantee instability; a rigorous proof of stability is still beyond our grasp.

The ansatz permits a centre manifold reduction, and the system can then be described in terms of a finite number of amplitude equations for the critical modes. These equations inherit much of the symmetry of the original partial differential equations, which greatly restricts their form, so the normal form equations can be written in terms of a small number of coefficients. The values of these coefficients are in general complicated expressions that depend upon the original chemical system and are found through the centre manifold reduction, but we make no assumptions in this paper about the specifics of the reaction-diffusion model. By assuming that any centre manifold reduction has already been performed and examining only the normal form, we thus study the Hopf bifurcation in a model-independent way. Our results are equally applicable to any generic Hopf pattern formation problem in three dimensions.

We assume henceforth that the system has the periodicity of a cubic lattice with wavevectors proportional to $(l, m, n)$ for integer $l, m$ and $n$. The symmetry of the system of amplitude equations is then determined by the choice of which wavevectors have the critical wavenumber $k_{\mathrm{c}}$, and hence correspond to critical modes. The different choices give infinitely many different representations of the relevant symmetry group. In this paper we restrict attention to two of the simplest representations, corresponding to the FCC and BCC lattices.

In section 2 we review the basic tools of equivariant theory as they relate to the Hopf bifurcation problem on the FCC lattice. We also find the most general possible normal form for the amplitude equations (up to cubic order in the amplitudes) that is consistent with the required symmetry. In section 3 we find the $\mathbb{C}$-axial solutions, along with their branching equations and stabilities. We solve this problem by first finding the solutions to a bifurcation problem with a larger symmetry group, then using them to determine the solutions on the FCC lattice. We find there are $15 \mathbb{C}$-axial solutions, which are generically guaranteed to have primary bifurcation branches, at least 12 of which can be stable for appropriate choices of the parameters. There are also certain subaxial isotropy subgroups on whose fixed point subspaces the system reduces to the problem studied in [18]. Thus for some parameter values there are primary branches of subaxial solutions, invariant tori corresponding to quasiperiodic solutions and indications of the possibility of chaos and bursting phenomena. In section 4 we study the Hopf bifurcation on the BCC lattice. The same technique allows us to find the $33 \mathbb{C}$-axial solution branches and their stability criteria. In section 5 we discuss how appropriate group extensions are chosen and the applicability of this method to other highly symmetric systems.

\section{Equivariance on the FCC lattice}

We first examine the Hopf bifurcation on the FCC lattice. We have eight critical wavevectors $\pm \boldsymbol{k}_{j}$ pointing to the vertices of a cube, with

$$
\begin{aligned}
\boldsymbol{k}_{1} & =\frac{k_{\mathrm{c}}}{\sqrt{3}}(1,1,1), & \boldsymbol{k}_{2} & =\frac{k_{\mathrm{c}}}{\sqrt{3}}(1,-1,-1), \\
\boldsymbol{k}_{3} & =\frac{k_{\mathrm{c}}}{\sqrt{3}}(-1,1,-1), & \boldsymbol{k}_{4} & =\frac{k_{\mathrm{c}}}{\sqrt{3}}(-1,-1,1) .
\end{aligned}
$$

A typical scalar field $\psi(x, y, z, t)$ (e.g. a concentration of one of the chemicals) can be described as a superposition of travelling waves in each of the eight directions:

$$
\psi(x, y, z, t)=\sum_{j}\left[z_{j}(t) \mathrm{e}^{\mathrm{i} k_{j} \cdot x}+w_{j}(t) \mathrm{e}^{-\mathrm{i} \boldsymbol{k}_{j} \cdot x}\right] \mathrm{e}^{-\mathrm{i} \omega t}+\text { c.c. }+ \text { h.o.t. },
$$


where 'c.c.' represents the complex conjugate and 'h.o.t.' represents higher order terms, spatial harmonics, which according to the centre manifold theorem [19] are quadratically small in the critical amplitudes $z_{j}$. The result of the centre manifold reduction is a set of evolution equations for the $z_{j}$ of the form $\dot{z}=\boldsymbol{F}(\boldsymbol{z})$, where we write the amplitudes as a vector

$$
z=\left(z_{1}, w_{1} ; z_{2}, w_{2} ; z_{3}, w_{3} ; z_{4}, w_{4}\right) \text {. }
$$

The function $\boldsymbol{F}(\boldsymbol{z})$ depends upon various model parameters, and we will distinguish one among them as the bifurcation parameter $\lambda$, writing $\boldsymbol{F}(z, \lambda)$ when necessary. We assume that as $\lambda$ increases through 0 the system undergoes a Hopf bifurcation. The amplitude equations inherit some symmetry from the original partial differential equations. Specifically, they must respect the symmetry group $G=T^{3} \rtimes \mathbb{O} \oplus \mathbb{Z}_{2}$, where $T^{3}$ is the three-torus of translations, (1) is the octahedral group of orientation-preserving symmetries of the cube and $\mathbb{Z}_{2}$ represents inversion through the origin. In the representation $\Gamma_{1}$ of $G$ appropriate to the FCC lattice, (1) contains all 24 permutations of the four $\left(z_{j}, w_{j}\right)$ pairs. For a translation by a vector $\boldsymbol{\alpha}$ the group element $\hat{\tau}_{\alpha} \in T^{3}$ acts on each $\left(z_{j}, w_{j}\right)$ pair by

$$
\hat{\tau}_{\alpha}:\left(z_{j}, w_{j}\right) \mapsto\left(z_{j} \mathrm{e}^{\mathrm{i} \boldsymbol{k}_{j} \cdot \boldsymbol{\alpha}}, w_{j} \mathrm{e}^{-\mathrm{i} \boldsymbol{k}_{j} \cdot \boldsymbol{\alpha}}\right),
$$

while $\hat{c} \in \mathbb{Z}_{2}$ acts by

$$
\hat{c}:\left(z_{j}, w_{j}\right) \mapsto\left(w_{j}, z_{j}\right) .
$$

$\hat{\tau}_{\alpha}$ and $\hat{c}$ resemble the phase shift and flip, respectively, of the usual action of $O(2)$ on each $\left(z_{j}, w_{j}\right)$ pair, but they act upon all four pairs simultaneously, so that the product of the phases $\prod_{j=1}^{4} \mathrm{e}^{\mathrm{i} k_{j} \cdot \alpha}$ is 1 and either all the pairs are flipped or none.

The centre manifold reduction can be done in such a way that the normal form has an additional $S^{1}$ symmetry [1], corresponding to translation in time, that acts by

$$
\hat{\phi} \cdot z=\mathrm{e}^{\mathrm{i} \phi} z
$$

Thus the symmetry of the reduced equations is $\tilde{\Gamma}_{1}=\Gamma_{1} \times S^{1}$.

If $z(t)$ is a solution then so is $\gamma \cdot z$ for any $\gamma \in \tilde{\Gamma}_{1}$. The normal form must therefore be $\tilde{\Gamma}_{1}$-equivariant, i.e.

$$
\gamma \cdot \boldsymbol{F}(\boldsymbol{z})=\boldsymbol{F}(\gamma \cdot z), \quad \forall \gamma \in \tilde{\Gamma}_{1}
$$

As we will see, this limits which terms can appear in the normal form.

\subsection{Normal forms}

We wish to do a model-independent analysis, and hence make no further assumptions about the system $\boldsymbol{F}$. In order to examine the dynamics of the system we use the most general possible $\tilde{\Gamma}_{1}$-equivariant Taylor expansion. To find it we employ a counting argument. The Poincaré series for the equivariants is defined by

$$
P(t) \equiv \frac{1}{\operatorname{Vol}\left(\tilde{\Gamma}_{1}\right)} \int_{\gamma \in \tilde{\Gamma}_{1}} \frac{\operatorname{Tr}(\gamma)}{\operatorname{det}[\mathbf{1}-t \gamma]},
$$

where the integral sign represents the sum over the discrete parts of the group and the integral over the continuous parts. As explained in [10,20-22], the coefficient of $t^{n}$ in the Taylor expansion of $P(t)$ is the number of linearly independent equivariant terms that are homogeneous of order $n$ in $z$. Thus $P(t)$ is a generating function for the number of equivariants at each order. For the representation of the group $\left(T^{3} \rtimes \mathbb{O} \oplus \mathbb{Z}_{2}\right) \times S^{1}$ appropriate to the FCC 
lattice, a straightforward calculation reveals that

$$
\begin{aligned}
P(t)= & {\left[t+2 t^{3}+11 t^{5}+34 t^{7}+76 t^{9}+145 t^{11}+230 t^{13}+280 t^{15}+289 t^{17}+244 t^{19}+158 t^{21}\right.} \\
& \left.+77 t^{23}+31 t^{25}+6 t^{27}\right]\left[\left(1-t^{2}\right)^{4}\left(1-t^{4}\right)^{4}\left(1-t^{6}\right)^{4}\right]^{-1} \\
= & t+6 t^{3}+33 t^{5}+146 t^{7}+\mathrm{O}\left(t^{9}\right) .
\end{aligned}
$$

We thus have exactly one linear, six cubic and 33 quintic equivariant terms. By rescaling $\lambda$, the third-order general system can thus be written

$$
\begin{aligned}
\dot{z}_{1}=(\lambda+\mathrm{i} \omega) z_{1} & +a\left|z_{1}\right|^{2} z_{1}+b\left(\left|z_{2}\right|^{2}+\left|z_{3}\right|^{2}+\left|z_{4}\right|^{2}\right) z_{1}+c\left|w_{1}\right|^{2} z_{1} \\
& +d\left(\left|w_{2}\right|^{2}+\left|w_{3}\right|^{2}+\left|w_{4}\right|^{2}\right) z_{1}+e\left(z_{2} w_{2}+z_{3} w_{3}+z_{4} w_{4}\right) \bar{w}_{1} \\
& +f\left(\bar{z}_{2} w_{3} w_{4}+\bar{z}_{3} w_{2} w_{4}+\bar{z}_{4} w_{2} w_{3}\right)
\end{aligned}
$$

for arbitrary real coefficients $\lambda, \omega$ and arbitrary complex coefficients $a, b, c, d, e$ and $f$. The equations for $\dot{w}_{1}, \dot{z}_{2}$, etc, are obtained from this through equivariance. This is a vast improvement over the 7752 independent complex coefficients that the general nonequivariant system possesses through third order. The coefficients in (4) are in general complicated functions of the parameters for any given model. However, the fact that we can reduce any appropriate system of PDEs to the above system with only eight coefficients allows us to make model-independent predictions.

\subsection{Isotropy subgroups}

The symmetry of the problem not only restricts the form of the amplitude equations but also guarantees the existence of certain solutions. The trivial solution $z=\mathbf{0}$ is the only solution with the full symmetry $\tilde{\Gamma}_{1}$; we characterize other solutions by the symmetry they have. We will need the following definitions from [1].

Definition. Given $z \in \mathbb{C}^{n}$, the isotropy subgroup of $z$ is

$$
\Sigma(z) \equiv\left\{\sigma \in \tilde{\Gamma}_{1}: \sigma \cdot z=z\right\}
$$

Definition. Given an isotropy subgroup $\Sigma \subseteq \tilde{\Gamma}_{1}$, its fixed point subspace is

$$
\operatorname{Fix}(\Sigma) \equiv\left\{z \in \mathbb{C}^{n}: \sigma \cdot z=z, \forall \sigma \in \Sigma\right\}
$$

An immediate consequence of these two definitions is the fact that if $\Sigma_{1} \subseteq \Sigma_{2}$ are both isotropy subgroups, then $\operatorname{Fix}\left(\Sigma_{2}\right) \subseteq \operatorname{Fix}\left(\Sigma_{1}\right)$. Furthermore, any fixed point subspace is an invariant subspace under the flow $\boldsymbol{F}(\boldsymbol{z})$. Thus the dynamics in a fixed point subspace $V$ can be analysed by examining the restriction $\left.\boldsymbol{F}\right|_{V}$.

Let $\gamma \in \tilde{\Gamma}_{1}$ and let $z$ be an equilibrium solution with isotropy subgroup $\Sigma$. By the physical symmetry of the problem, $\gamma \cdot z$ is also an equilibrium solution with isotropy subgroup $\gamma \Sigma \gamma^{-1}$ conjugate to $\Sigma$. We consider these solutions to be equivalent, and likewise consider two isotropy subgroups to be equivalent if they are conjugate to one another.

We will need two more definitions from [1].

Definition. A representation $\Gamma_{1}$ is absolutely irreducible if the only matrices that commute with all elements of $\Gamma_{1}$ are scalar multiples of the identity.

Definition. The space $\mathbb{R}^{2 n}=\mathbb{C}^{n}$ is $\Gamma_{1}$-simple if either

(i) $\Gamma_{1}$ acts irreducibly but not absolutely irreducibly on $\mathbb{R}^{2 n}$, or

(ii) $\Gamma_{1}$ acts on $\mathbb{R}^{n} \oplus \mathbb{R}^{n}$, where the action on $\mathbb{R}^{n}$ is absolutely irreducible. 
The usefulness of these definitions stems from the fact that the generic $\Gamma_{1}$-equivariant Hopf bifurcation problem is $\Gamma_{1}$-simple [1], and from the following theorem.

Equivariant Hopf theorem (EHT) [1]. Let $\mathbb{C}^{n}$ be $\Gamma_{1}$-simple, let $\tilde{\Gamma}_{1}=\Gamma_{1} \times S^{1}$ and let the system $\boldsymbol{F}(z, \lambda): \mathbb{C}^{n} \times \mathbb{R} \mapsto \mathbb{C}^{n}$ be a $\tilde{\Gamma}_{1}$-equivariant system of ODEs with

$$
(\mathrm{d} \boldsymbol{F})_{\mathbf{0}, 0}=\left(\begin{array}{rr}
\mathbf{0} & -\mathbf{1} \\
\mathbf{1} & \mathbf{0}
\end{array}\right) \omega .
$$

Assume that as $\lambda$ increases through 0 the eigenvalues pass through the imaginary axis with nonzero speed. Let $\Sigma \subset \tilde{\Gamma}_{1}$ be an isotropy subgroup of spatiotemporal symmetries satisfying $\operatorname{dim}(\operatorname{Fix}(\Sigma))=2$. Then there exists a unique smooth branch of small-amplitude periodic solutions to $\dot{z}=\boldsymbol{F}(z)$ with period near $2 \pi / \omega$, having $\Sigma$ as their isotropy subgroup.

Isotropy subgroups with two-dimensional fixed point subspace are termed $\mathbb{C}$-axial, and they are guaranteed by the EHT to have primary solution branches.

\subsection{Stability}

The stabilities of the resulting solutions are determined by the linearization matrix $\mathrm{d} \boldsymbol{F}$, evaluated on the solution branch. The presence of any positive eigenvalues guarantees that the solution is unstable. We cannot require, however, that all the eigenvalues be negative, as equivariance guarantees a certain number of zero eigenvalues. Consider a solution of the form $z=(A, A ; 0,0 ; 0,0 ; 0,0)$ with $A$ real, which corresponds to lamellae that oscillate in magnitude, i.e.

$$
\psi(\boldsymbol{x}, t)=\left(A \mathrm{e}^{\mathrm{i} \boldsymbol{k}_{1} \cdot \boldsymbol{x}}+A \mathrm{e}^{-\mathrm{i} \boldsymbol{k}_{1} \cdot \boldsymbol{x}}\right) \mathrm{e}^{-\mathrm{i} \omega t}+\text { c.c. }+ \text { h.o.t. } \approx 4 A \cos \left(\boldsymbol{k}_{1} \cdot \boldsymbol{x}\right) \cos (\omega t) .
$$

An infinitesimal change in $z$ in the direction of $(\mathrm{i},-\mathrm{i} ; 0,0 ; 0,0 ; 0,0)$ corresponds to a small translation of the pattern in the direction of $\boldsymbol{k}_{1}$. By the translational symmetry of the problem this is still a solution. In fact, there is a whole one-dimensional orbit of solutions, related by translations, and thus the stability eigenvalue in that direction must vanish. This is true for any nontrivial translation of the pattern. In general we expect $\operatorname{dim}\left(\tilde{\Gamma}_{1}\right)-\operatorname{dim}(\Sigma)=4-\operatorname{dim}(\Sigma)$ zero eigenvalues. In the null eigenspace the dynamics are trivial, so these zero eigenvalues do not spoil the stability. If all the other eigenvalues of $\mathrm{d} \boldsymbol{F}$ are negative then, following [1], we call the solution linearly orbitally stable.

\section{Solutions on the FCC lattice}

We now turn to the main focus of this paper: finding the solutions to a bifurcation problem with large symmetry group and high dimensionality. We will accomplish this by extending the symmetry group to a still larger one. For the FCC lattice we consider not only the $\mathbb{C}$-axial solutions, but also some of the subaxial solutions (those whose isotropy subgroups have fixed point subspaces of dimensionality $>2$ ), some of which can have primary bifurcations.

\section{1. $\mathbb{C}$-axial solutions}

The determination of the $\mathbb{C}$-axial isotropy subgroups of a given group $\tilde{\Gamma}_{1}$ is often a very involved process. In analysing the Hopf bifurcation on planar lattices [3] first finds all the subgroups of the holohedry (in our case this would be $\mathbb{O} \oplus \mathbb{Z}_{2}$ ). From these all the shifted subgroups $K \subset \Gamma_{1}$ (subgroups containing no nontrivial translation) are found. For each of these is then found all subgroups $H$ such that $K \subset H \subset N_{\Gamma_{1}}(K)$ and $H / K$ is a Lie subgroup of $S^{1}$ and $H / K$ is a maximal Abelian subgroup of $N_{\Gamma_{1}}(K) / K$. (Here $N_{\Gamma_{1}}(K)$ is the normalizer of $K$ in $\Gamma_{1}$.) 
From each such pair $(H, K)$ a twisted subgroup is then formed and analysed to determine whether it is $\mathbb{C}$-axial. For a symmetry group representation as complicated as that for the FCC lattice (or the still more complicated representation for the BCC lattice, which we will study in section 4 ), the task of guaranteeing that we have found all the $\mathbb{C}$-axial subgroups is a daunting one. Fortunately, there is a simpler process $[13,14]$.

Consider two groups $G_{1} \subset G_{2}$ acting on the same vector space $V$ by representations $\tilde{\Gamma}_{1} \subset \tilde{\Gamma}_{2}$, respectively. Suppose we wish to find the $\mathbb{C}$-axial subgroups of $\tilde{\Gamma}_{1}$, but that it is easy to find the $\mathbb{C}$-axial subgroups of $\tilde{\Gamma}_{2}$. Let $\Sigma_{1} \subset \tilde{\Gamma}_{1}$ be an isotropy subgroup and let $V_{1}=\operatorname{Fix}\left(\Sigma_{1}\right)$ be its fixed point subspace. We extend $\Sigma_{1}$ to

$$
\Sigma_{2}=\left\{\sigma \in \tilde{\Gamma}_{2}: \sigma \cdot z=z, \forall z \in V_{1}\right\}
$$

and then let $V_{2}=\operatorname{Fix}\left(\Sigma_{2}\right)$. By construction $\Sigma_{1} \subseteq \Sigma_{2}$, so $V_{2} \subseteq V_{1}$. Also by construction $V_{1}$ is fixed under $\Sigma_{2}$, so $V_{1} \subseteq V_{2}$, and therefore $V_{1}=V_{2}$. We thus have the following fact.

Fact. The fixed point subspace of any isotropy subgroup of $\tilde{\Gamma}_{1}$ is the fixed point subspace of some isotropy subgroup of $\tilde{\Gamma}_{2}$.

Thus to find the isotropy subgroups of $\tilde{\Gamma}_{1}$ it is sufficient to find and analyse the isotropy subgroups of $\tilde{\Gamma}_{2}$. In fact, we can say more: every $\mathbb{C}$-axial solution under $\tilde{\Gamma}_{1}$ is a $\mathbb{C}$-axial solution under $\tilde{\Gamma}_{2}$. In order to find all the $\mathbb{C}$-axial solutions under $\tilde{\Gamma}_{1}$ we expand to a larger but (we hope) easier group $\tilde{\Gamma}_{2}$. We then find the $\mathbb{C}$-axial solutions under $\tilde{\Gamma}_{2}$ and use them to produce the $\mathbb{C}$-axial solutions under $\tilde{\Gamma}_{1}$.

The converse to our fact is not true. A $\tilde{\Gamma}_{2}$-solution $z$ corresponds to no $\tilde{\Gamma}_{1}$-solution if $\boldsymbol{F}(\boldsymbol{z})$ contains a $\tilde{\Gamma}_{1}$-equivariant (and $\tilde{\Gamma}_{2}$-nonequivariant) term under which $\operatorname{Fix}\left(\Sigma_{2}\right)$ is not an invariant subspace. More generally, we get no $\tilde{\Gamma}_{1}$-solution if there is a group element $\sigma \in \Sigma_{2} \backslash \tilde{\Gamma}_{1}$ which is necessary in order to pin down $\operatorname{Fix}\left(\Sigma_{2}\right)$ completely. A $\tilde{\Gamma}_{2}$-solution can also correspond to more than one $\tilde{\Gamma}_{1}$-solution. Given two different (i.e. nonconjugate) isotropy subgroups of $\tilde{\Gamma}_{1}$, their extensions can be conjugate to one another by some element $\gamma \in \tilde{\Gamma}_{2} \backslash \tilde{\Gamma}_{1}$.

For the FCC lattice we have $\tilde{\Gamma}_{1}=\left(T^{3} \rtimes \mathbb{O} \oplus \mathbb{Z}_{2}\right) \times S^{1}$. We enlarge this to $\tilde{\Gamma}_{2}$ by adding the generators $\hat{\theta}$ and $\hat{r}$, which act by

$$
\begin{aligned}
& \hat{\theta}: z_{j} \mapsto \mathrm{e}^{\mathrm{i} \theta} z_{j}, \quad \hat{\theta}: w_{j} \mapsto \mathrm{e}^{-\mathrm{i} \theta} w_{j}, \\
& \hat{r}:\left(z_{1}, w_{1} ; z_{2}, w_{2} ; z_{3}, w_{3} ; z_{4}, w_{4}\right) \mapsto\left(w_{1}, z_{1} ; z_{2}, w_{2} ; z_{3}, w_{3} ; z_{4}, w_{4}\right) .
\end{aligned}
$$

These, together with the permutations from $\mathbb{O}$, allow us full liberty to phase shift and/or flip each $\left(z_{j}, w_{j}\right)$ pair independently. That is, we have an $O(2)$ action of translations and reflections on each pair $\left(z_{j}, w_{j}\right)$, and we retain our full permutation group $S_{4}$ among the pairs. We now have four independent translations and reflections, so we have in effect bent the four wavevectors until they are mutually orthogonal. We thus have the symmetry group of the Hopf bifurcation on the simple hypercubic lattice in four spatial dimensions.

Our enlarged group $\tilde{\Gamma}_{2}$ can also be written in terms of the wreath product as $\tilde{\Gamma}_{2}=$ $\left[O(2)<S_{4}\right] \times S^{1}$. The wreath product is defined in $[12,23,24]$ and is used to describe a system of identical subsystems. Suppose each subsystem has the local symmetry group $L$ and the whole system is symmetric under the global group $G$ of permutations of the subsystems. If each $L$ acting on each subsystem is allowed to act independently then the whole group of symmetries is the wreath product $L$ ? $G$.

In [12] a method is presented to find all the $\mathbb{C}$-axial isotropy subgroups of $\left(L_{2} G\right) \times S^{1}$, given the $\mathbb{C}$-axial isotropy subgroups of $L \times S^{1}$. For our case $L=O(2)$ and we need the isotropy subgroups of the action of $O(2) \times S^{1}$ on each $\left(z_{j}, w_{j}\right)$ pair. This is the Hopf bifurcation problem with $O(2)$ symmetry and the solutions are well-known. Representative $\mathbb{C}$-axial solutions are of the form $(x, x)$ and $(x, 0)$, for $x \in \mathbb{C}$. 
The ease with which the $\mathbb{C}$-axial solutions of $\left(L_{2} G\right) \times S^{1}$ can be constructed by this method is best illustrated with the equivalent steady-state problem of [24]. To find the axial isotropy subgroups of $L$; $G$ we first define a block to be a set of subsystems on which some subgroup $H \subseteq G$ acts transitively. Given a block and an axial solution of $L$, we repeat that solution in each subsystem of the block, and set the amplitudes in the other subsystems to 0 . For all the examples studied in this paper the global group is $G=S_{n}$ for some $n$, so we need merely copy the axial solution of $L$ into 1, 2, 3, etc, subsystems. For the Hopf bifurcation problem of [12] the blocks are further subdivided into subblocks, and certain phases multiply the repetitions of the axial solutions of $L$, but the method is not significantly more difficult to employ.

Following this method, we do a calculation similar to that done in [23] for the simple cubic lattice. The result is the $12 \mathbb{C}$-axial solutions on the simple hypercubic lattice. Typical elements of the fixed point subspaces of the $\mathbb{C}$-axial isotropy subgroups of $\tilde{\Gamma}_{2}$ are listed in table 1.

To find the $\mathbb{C}$-axial solutions of the original group $\tilde{\Gamma}_{1}$ we take each $\mathbb{C}$-axial solution $z$, construct $\gamma \cdot z$ for an arbitrary $\gamma \in \tilde{\Gamma}_{2} \backslash \tilde{\Gamma}_{1}$ and let $\Sigma_{1}(\gamma \cdot z)$ be its isotropy subgroup in $\tilde{\Gamma}_{1}$. We then solve $\operatorname{dim}\left(\operatorname{Fix}\left(\Sigma_{1}(\gamma \cdot z)\right)\right)=2$ for $\gamma$. In practice the possibilities are most effectively winnowed by first exploiting the normal form. Fortunately, we have used only two generators to enlarge the group, so this is a relatively easy calculation. Consider, for instance, the standing tesseracts. The flip operator $\hat{r}$ does nothing to this solution, so we need only consider the phase operator $\hat{\theta}$. Any solution based on the standing tesseracts can be translated to one with no phase in the first three subsystems, so we need only consider solutions of the form $(x, x ; x, x ; x, x ; x v, x / v)$, for some complex $v$ of unit modulus. Substituting this into the normal form (4), we can easily verify that this subspace is only invariant for $v= \pm 1$. Thus the only possibilities are the standing fcc (SFCC) $(v=1)$ and standing double diamond (SDD) $(v=-1)$ solutions. Of course, invariance under the cubic truncation does not guarantee that a solution is $\mathbb{C}$-axial, so each of these solutions must then be checked. To do this we need merely confirm that, e.g., the SFCC solution has enough symmetries to restrict any solution to be of the form SFCC.

Table 1. Elements of the fixed point subspaces of the $\mathbb{C}$-axial subgroups of $\tilde{\Gamma}_{2}=\left[O(2) \gtrless S_{4}\right] \times S^{1}$ and of $\tilde{\Gamma}_{1}$, which is the FCC representation of $T^{3} \rtimes \mathbb{O} \oplus \mathbb{Z}_{2}$. Here $x \in \mathbb{C}, \xi=\mathrm{e}^{\mathrm{i} \pi / 3}$ and $\chi=\mathrm{e}^{\mathrm{i} \pi / 4}$. The full names of the $\tilde{\Gamma}_{1}$-solutions are given in table 2 .

\begin{tabular}{llll}
\hline$\tilde{\Gamma}_{2}$ name & $\tilde{\Gamma}_{2}$-solution $z$ & $\tilde{\Gamma}_{1}$-solution $z$ & $\tilde{\Gamma}_{1}$ name \\
\hline Standing lamellae (SL) & $x(1,1 ; 0,0 ; 0,0 ; 0,0)$ & $x(1,1 ; 0,0 ; 0,0 ; 0,0)$ & SL \\
Standing squares (SS) & $x(1,1 ; 1,1 ; 0,0 ; 0,0)$ & $x(1,1 ; 1,1 ; 0,0 ; 0,0)$ & SR \\
Alternating lamellae (AL) & $x(1,1 ; \mathrm{i}, \mathrm{i} ; 0,0 ; 0,0)$ & $x(1,1 ; \mathrm{i}, \mathrm{i} ; 0,0 ; 0,0)$ & $\mathrm{AL}$ \\
Standing cubes (SC) & $x(1,1 ; 1,1 ; 1,1 ; 0,0)$ & & $\mathrm{CR}$ \\
Cycling squares (CS) & $x\left(1,1 ; \xi, \xi ; \xi^{2}, \xi^{2} ; 0,0\right)$ & $x\left(1,1 ; \xi, \xi ; \xi^{2}, \xi \xi^{2} ; 0,0\right)$ & SFCC \\
Standing tesseracts (ST) & $x(1,1 ; 1,1 ; 1,1 ; 1,1)$ & $x(1,1 ; 1,1 ; 1,1 ; 1,1)$ & SDD \\
& & $x(1,1 ; 1,1 ; 1,1 ;-1,-1)$ & AR1 \\
Alternating squares (AS) & $x(1,1 ; 1,1 ; \mathrm{i}, \mathrm{i} ; \mathrm{i}, \mathrm{i})$ & $x(1,1 ; 1,1 ; \mathrm{i}, \mathrm{i} ; \mathrm{i})$ & $\mathrm{AR2}$ \\
& & $x(1,1 ; 1,1 ; \mathrm{i}, \mathrm{i} ;-\mathrm{i},-\mathrm{i})$ & $\mathrm{TL}$ \\
Cycling (distorted) cubes (CC) & $x\left(1,1 ; \chi, \chi ; \chi^{2}, \chi^{2} ; \chi^{3}, \chi^{3}\right)$ & & TR1 \\
Travelling lamellae (TL) & $x(1,0 ; 0,0 ; 0,0 ; 0,0)$ & $x(1,0 ; 0,0 ; 0,0 ; 0,0)$ & TR2 \\
Travelling squares (TS) & $x(1,0 ; 1,0 ; 0,0 ; 0,0)$ & $x(1,0 ; 1,0 ; 0,0 ; 0,0)$ & TP \\
Travelling cubes (TC) & $x(1,0 ; 1,0 ; 1,0 ; 0,0)$ & $x(1,0 ; 0,1 ; 0,0 ; 0,0)$ & AFD \\
Travelling tesseracts (TT) & $x(1,0 ; 1,0 ; 1,0 ; 1,0)$ & $x(1,0 ; 1,0 ; 1,0 ; 0,0)$ & TFCC \\
& & $x(1,0 ; 1,0 ; 0,1 ; 0,1)$ & TDD
\end{tabular}


The resulting 15 solutions are shown in table 1 . The standing tesseract solution corresponds to these two solutions on the FCC lattice: the SFCC and SDD solutions. They are the standing wave equivalents of the steady-state fcc and double diamond (dd) solutions found in [9]. The dd solution has maxima located at the vertices of a diamond lattice and minima at the vertices of another, interlocking diamond lattice. As we have just seen, these two solutions are conjugate in the extended symmetry group by an overall phase shift that is unavailable in the smaller symmetry group.

Similarly, the standing lamellae (SL) and standing rhombic prism (SR) solutions are oscillatory versions of their steady-state equivalents. The alternating lamellae (AL) solution oscillates between lamellae aligned with $\boldsymbol{k}_{1}$ and lamellae aligned with $\boldsymbol{k}_{2}$.

The cycling rhomb $(\mathrm{CR})$ solution cycles through rhombic prism patterns with three different orientations. At time $t=\pi / 6$ it resembles rhombic prisms with wavevectors $k_{1}$ and $\boldsymbol{k}_{3}$. A time $\pi / 3$ later it resembles rhombs with wavevectors $\boldsymbol{k}_{2}$ and $\boldsymbol{k}_{3}$, while another $\pi / 3$ later the wavevectors are $\boldsymbol{k}_{1}$ and $\boldsymbol{k}_{2}$.

The alternating squares on the hypercubic lattice give us two different solutions of alternating rhombs. At time $t=0$ both AR1 and AR2 resemble rhombic prisms with wavevectors $\boldsymbol{k}_{1}$ and $\boldsymbol{k}_{2}$. At time $t=\pi / 2$ they both resemble rhombic prisms with wavevectors $\boldsymbol{k}_{3}$ and $\boldsymbol{k}_{4}$. The difference lies in the relative spatial displacement between the two sets of rhombs. At time $t=0$ the scalar field $\psi$ takes the approximate form

$$
\psi(\boldsymbol{x}, 0) \propto \cos \left(\boldsymbol{k}_{1} \cdot \boldsymbol{x}\right)+\cos \left(\boldsymbol{k}_{2} \cdot \boldsymbol{x}\right),
$$

which has maxima along the lines given by $x=m \pi, y+z=n \pi$ for $m$ and $n$ any two integers of the same parity. At time $t=\pi / 2$ the scalar field $\psi$ takes the approximate form

$$
\psi\left(\boldsymbol{x}, \frac{\pi}{2}\right) \propto \cos \left(\boldsymbol{k}_{3} \cdot \boldsymbol{x}\right) \pm \cos \left(\boldsymbol{k}_{4} \cdot \boldsymbol{x}\right)
$$

with + for AR1 and - for AR2. For AR1 the maxima are along the lines given by $x=m \pi$ and $y-z=n \pi$, while for AR2 the maxima are along the lines given by $x=\left(m+\frac{1}{2}\right) \pi$ and $y-z=\left(n+\frac{1}{2}\right) \pi$. (In both cases $m$ and $n$ must again be of the same parity.) Thus, for instance, the locus of maxima at $t=0$ intersects the locus of maxima at $t=\pi / 2$ for AR 1 , but not for AR2.

Most of the solutions resulting from travelling patterns on the hypercubic lattice are themselves travelling patterns. The travelling lamellae (TL) are the same on the FCC lattice, while the travelling cubes (TC) are distorted into travelling parallelopipeds (TP). The travelling squares (TS) produce two inequivalent sets of travelling rhombs: TR1 with wavevectors $\boldsymbol{k}_{1}$ and $\boldsymbol{k}_{2}$ and TR2 with wavevectors $\boldsymbol{k}_{1}$ and $-\boldsymbol{k}_{2}$. These have the same spatial structure, but travel in different directions. The travelling tesseracts correspond to three solutions: the travelling fcc (TFCC) and travelling dd (TDD) solutions and a solution that alternates between the fcc and dd structures (AFD).

Lastly, note that the standing and cycling cube solutions correspond to no $\mathbb{C}$-axial solutions at all on the FCC lattice. For both of these it is the term with coefficient $f$ in the normal form that breaks the invariance of these subspaces.

These solutions are all guaranteed by the EHT to bifurcate directly from the trivial solution at $\lambda=0$. We can easily find the branching equations for each of these isotropy subgroups by restricting the system (4) to the relevant fixed point subspace. These equations are listed in table 2, together with the number of group-theoretically guaranteed zero eigenvalues.

We have also calculated the stabilities of these solutions in terms of the normal form coefficients $a, \ldots, f$. No solution has more null eigenvalues than are required by equivariance, so the linear orbital stabilities are generically determined at cubic order. Stability criteria for each solution are listed in table 3. The solutions TR1 and TP can never be stable, and we do 
Table 2. Solution branches for the $\mathbb{C}$-axial solutions in terms of the coefficients of the normal form (4). The subscript $r$ denotes the real part. The column labelled \# is the number of grouptheoretically guaranteed zero eigenvalues, and is equal to $4-\operatorname{dim}(\Sigma)$.

\begin{tabular}{lll}
\hline Name & $\#$ & Branching equation \\
\hline SL (standing lamellae) & 2 & $\lambda=-\left(a_{\mathrm{r}}+c_{\mathrm{r}}\right)|x|^{2}$ \\
SR (standing rhombs) & 3 & $\lambda=-\left(a_{\mathrm{r}}+b_{\mathrm{r}}+c_{\mathrm{r}}+d_{\mathrm{r}}+e_{\mathrm{r}}\right)|x|^{2}$ \\
AL (alternating lamellae) & 3 & $\lambda=-\left(a_{\mathrm{r}}+b_{\mathrm{r}}+c_{\mathrm{r}}+d_{\mathrm{r}}-e_{\mathrm{r}}\right)|x|^{2}$ \\
CR (cycling rhombs) & 4 & $\lambda=-\left(a_{\mathrm{r}}+2 b_{\mathrm{r}}+c_{\mathrm{r}}+2 d_{\mathrm{r}}-e_{\mathrm{r}}\right)|x|^{2}$ \\
SFCC (standing fcc) & 4 & $\lambda=-\left(a_{\mathrm{r}}+3 b_{\mathrm{r}}+c_{\mathrm{r}}+3 d_{\mathrm{r}}+3 e_{\mathrm{r}}+3 f_{\mathrm{r}}\right)|x|^{2}$ \\
SDD (standing double diamond) & 4 & $\lambda=-\left(a_{\mathrm{r}}+3 b_{\mathrm{r}}+c_{\mathrm{r}}+3 d_{\mathrm{r}}+3 e_{\mathrm{r}}-3 f_{\mathrm{r}}\right)|x|^{2}$ \\
AR1 (alternating rhombs 1) & 4 & $\lambda=-\left(a_{\mathrm{r}}+3 b_{\mathrm{r}}+c_{\mathrm{r}}+3 d_{\mathrm{r}}-e_{\mathrm{r}}+f_{\mathrm{r}}\right)|x|^{2}$ \\
AR2 (alternating rhombs 2) & 4 & $\lambda=-\left(a_{\mathrm{r}}+3 b_{\mathrm{r}}+c_{\mathrm{r}}+3 d_{\mathrm{r}}-e_{\mathrm{r}}-f_{\mathrm{r}}\right)|x|^{2}$ \\
TL (travelling lamellae) & 1 & $\lambda=-a_{\mathrm{r}}|x|^{2}$ \\
TR1 (travelling rhombs 1) & 2 & $\lambda=-\left(a_{\mathrm{r}}+b_{\mathrm{r}}\right)|x|^{2}$ \\
TR2 (travelling rhombs 2) & 2 & $\lambda=-\left(a_{\mathrm{r}}+d_{\mathrm{r}}\right)|x|^{2}$ \\
TP (travelling parallelopipeds) & 3 & $\lambda=-\left(a_{\mathrm{r}}+2 b_{\mathrm{r}}\right)|x|^{2}$ \\
AFD (alternating fcc/dd) & 4 & $\lambda=-\left(a_{\mathrm{r}}+3 b_{\mathrm{r}}\right)|x|^{2}$ \\
TFCC (travelling fcc) & 3 & $\lambda=-\left(a_{\mathrm{r}}+b_{\mathrm{r}}+2 d_{\mathrm{r}}+f_{\mathrm{r}}\right)|x|^{2}$ \\
TDD (travelling dd) & 3 & $\lambda=-\left(a_{\mathrm{r}}+b_{\mathrm{r}}+2 d_{\mathrm{r}}-f_{\mathrm{r}}\right)|x|^{2}$ \\
\hline
\end{tabular}

not know whether the CR solution can be stable. However, each of the 12 other solutions can be orbitally asymptotically stable for appropriate values of the coefficients.

\subsection{Subaxial isotropy: Swiftian solutions}

In addition to the $\mathbb{C}$-axial solutions guaranteed by the EHT, certain submaximal solutions are also guaranteed to have primary bifurcations in open regions of parameter space. We can use the method of [23] to find solutions with four-dimensional fixed point subspace in $\tilde{\Gamma}_{2}$ and then see what solutions they give us in $\tilde{\Gamma}_{1}$. For example, some of the four-dimensional fixed point subspaces are listed in table 4.

Consider the second fixed point subspace, $V$, with typical element

$$
z=(x, x ; y, y ; 0,0 ; 0,0) .
$$

As $V$ is an invariant subspace under the flow of $\boldsymbol{F}$, the dynamics in $V$ are determined by $\left.\boldsymbol{F}\right|_{V}$, which, to cubic order, is given by

$$
\begin{aligned}
& \dot{x}=(\lambda+\mathrm{i} \omega) x+(a+c)|x|^{2} x+(b+d)|y|^{2} x+e \bar{x} y^{2}, \\
& \dot{y}=(\lambda+\mathrm{i} \omega) y+(a+c)|y|^{2} y+(b+d)|x|^{2} y+e x^{2} \bar{y} .
\end{aligned}
$$

This system inherits some of the symmetry $\tilde{\Gamma}_{1}$. Specifically, if $N(\Sigma)$ is the normalizer of $\Sigma$ in $\tilde{\Gamma}_{1}$ then the restricted system is equivariant under the group $N(\Sigma) / \Sigma$. For this particular example it is easy to see that the restricted system is equivariant under the symmetry group $\mathbb{D}_{4} \times S^{1}$. Here $\mathbb{D}_{4}$ is the symmetry of the square, generated by $(x, y) \mapsto(-x, y)$ and $(x, y) \mapsto(y, x)$. The former corresponds to a translation by an amount $(2 \pi, \pi, \pi) / 4 k_{\mathrm{c}}$, while the latter is one of the permutation symmetries of $\mathbb{O}$.

On each of the invariant subspaces in table 4 the restricted system is a Hopf bifurcation with $\mathbb{D}_{4}$ symmetry. The general such system, to third order, can be written

$$
\begin{aligned}
& \dot{x}=\left[\lambda+\mathrm{i} \omega+A\left(|x|^{2}+|y|^{2}\right)+B|x|^{2}\right] x+C \bar{x} y^{2}, \\
& \dot{y}=\left[\lambda+\mathrm{i} \omega+A\left(|x|^{2}+|y|^{2}\right)+B|y|^{2}\right] y+C x^{2} \bar{y}
\end{aligned}
$$


Table 3. Stability criteria for the $\mathbb{C}$-axial solutions on the FCC lattice. The subscript $\mathrm{r}$ (i) denotes the real (imaginary) part. The solution is orbitally asymptotically stable if each of the terms shown is negative.

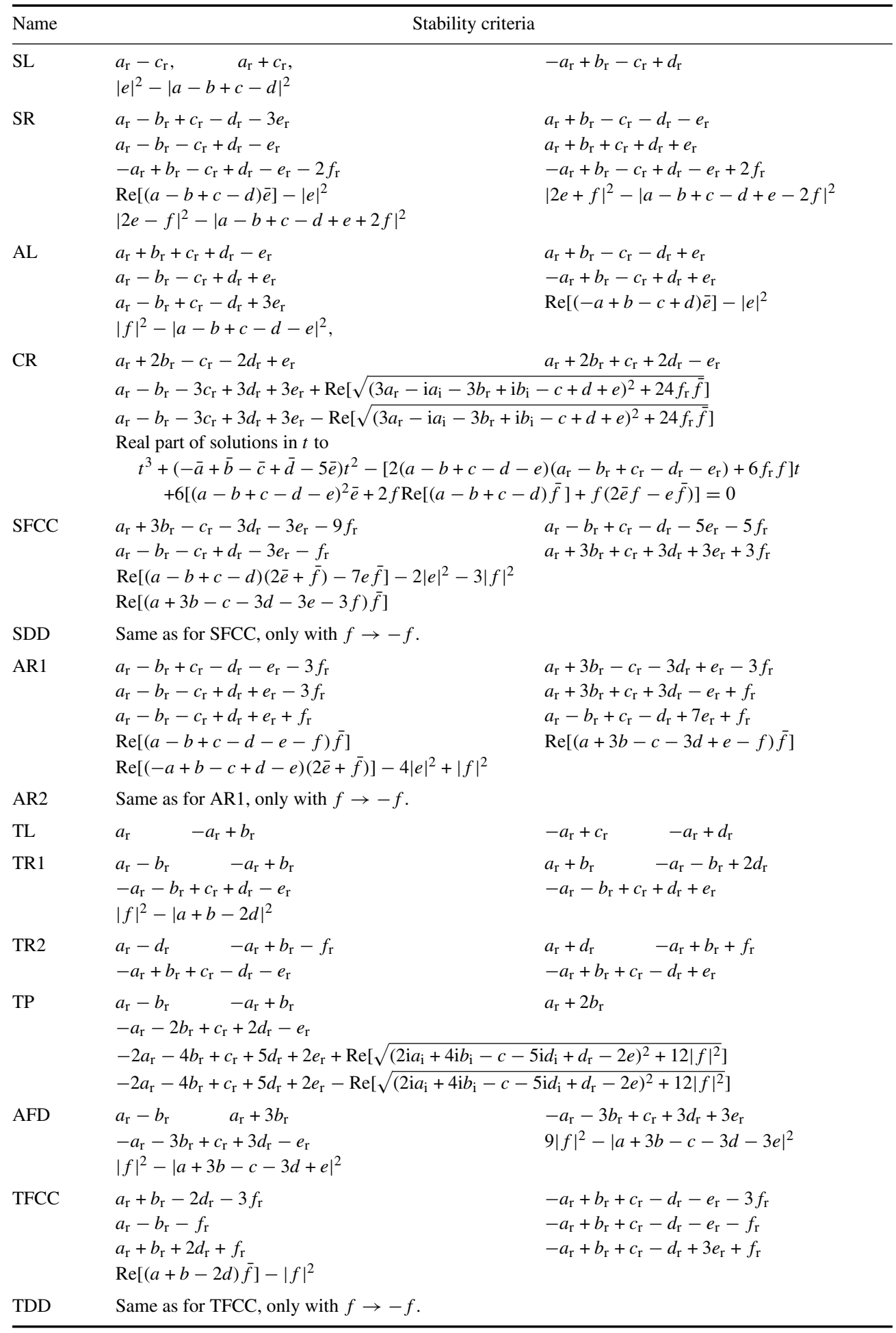


Table 4. Elements of some four-dimensional fixed point subspaces of $\tilde{\Gamma}_{1}$ for the FCC lattice. Here again $\xi=\mathrm{e}^{\mathrm{i} \pi / 3}$ and $x, y \in \mathbb{C}$.

\begin{tabular}{llll}
\hline Name & $z \in \mathrm{Fix}(\Sigma)$ & $B$ & $C$ \\
\hline 1 & $(x, y ; x, y ; x, y ; x, y)$ & $a+3 b-c-3 d-3 e$ & $3 f$ \\
2 & $(x, x ; y, y ; \mathrm{i} x, \mathrm{i} x ; \mathrm{i} y, \mathrm{i} y)$ & $a-b+c-d-e$ & $f$ \\
3 & $(x, x ; y, y ; 0,0 ; 0,0)$ & $a-b+c-d$ & $e$ \\
4 & $(x, x ; x, x ; y, y ; y, y)$ & $a-b+c-d+e-2 f$ & $2 e+f$ \\
5 & $(x, 0 ; x, 0 ; 0, y ; 0, y)$ & $a+b-2 d$ & $f$ \\
6 & $(x, 0 ; y, 0 ; 0,0 ; 0,0)$ & $a-b$ & 0 \\
7 & $(x, 0 ; 0, y ; 0,0 ; 0,0)$ & $a-d$ & 0 \\
8 & $(x, 0 ; x, 0 ; y, 0 ; y, 0)$ & $a-b$ & 0 \\
9 & $(x, 0 ; 0, x ; y, 0 ; 0, y)$ & $a-b-d$ & 0 \\
10 & $(x, y ; 0,0 ; 0,0 ; 0,0)$ & $a-c$ & 0 \\
11 & $(x, y ; x, y ; 0,0 ; 0,0)$ & $a+b-c-d-e$ & 0 \\
12 & $(x, y ; y, x ; 0,0 ; 0,0)$ & $a-b-c+d-e$ & 0 \\
13 & $(x, y ; \mathrm{i} x, \mathrm{i} y ; 0,0 ; 0,0)$ & $a+b-c-d+e$ & 0 \\
14 & $(x, y ; \mathrm{i} y, \mathrm{i} x ; 0,0 ; 0,0)$ & $a-b-c+d+e$ & 0 \\
15 & $\left(x, y ; \xi x, \xi y ; \xi^{2} x, \xi^{2} y ; 0,0\right)$ & $a+2 b-c-2 d+e$ & 0 \\
\hline
\end{tabular}

with $A, B$ and $C$ arbitrary complex coefficients. This system was analysed by Swift [18], who found a primary branch of periodic subaxial solutions $(0 \neq x \neq y \neq 0)$ that exists precisely when

$$
|B|^{2}>|C|^{2}>|\operatorname{Re}(B \bar{C})| \text {. }
$$

For each of the subspaces in table 4 we have listed the parameters $B$ and $C$ of the restricted system; clearly the primary subaxial branches are possible for solutions $1-5$ and are impossible for solutions 6-15. These solutions cannot be stable even in the invariant subspace, let alone in the whole space $\mathbb{C}^{8}$.

Swift found invariant tori corresponding to quasiperiodic solutions when $|B|^{2}<|C|^{2}$ and $\operatorname{Re}[B]>3|\operatorname{Re}[C]|$. We thus expect such solutions for the Hopf bifurcation on the FCC lattice as well. For the right parameter values they can even be asymptotically stable within the invariant subspace. Swift also found evidence for chaotic dynamics, and we therefore expect it, too, on the FCC lattice.

Such subaxial solutions have been previously found for the Hopf bifurcations on the square [25] and simple cubic [12] lattices. They are likewise discovered through a consideration of a four-dimensional invariant subspace, on which the reduced system is equivariant under $\mathbb{D}_{4}$. For the FCC lattice, however, we have the possibility of yet richer dynamics. The reflection symmetry $(x, y) \mapsto(-x, y)$ of equation (5) is inherited directly from a spatial translation in $\Gamma_{1}=T^{3} \rtimes \mathbb{O} \oplus \mathbb{Z}_{2}$, and is an exact symmetry for solution 2 . Consider instead subspace 1 from table 4 , with typical element

$$
z=(x, y ; x, y ; x, y ; x, y) .
$$

This subspace too has the reflection $(x, y) \mapsto(-x, y)$, but it corresponds to translating in the $-k_{1}$ direction through $\frac{3}{4}$ of a wavelength, followed by waiting in time for $\frac{1}{4}$ of a period. More exactly, it is composed of the translation $\hat{\tau} \in T^{3}$ given by

$$
\hat{\tau}:\left(z_{1}, w_{1} ; z_{2}, w_{2} ; z_{3}, w_{3} ; z_{4}, w_{4}\right) \mapsto\left(\mathrm{i} z_{1},-\mathrm{i} w_{1} ; \mathrm{i} z_{2},-\mathrm{i} w_{2} ; \mathrm{i} z_{3},-\mathrm{i} w_{3} ; \mathrm{i} z_{4},-\mathrm{i} w_{4}\right),
$$

followed by the time translation $z \mapsto \mathrm{i} z$. The $S^{1}$ is only a normal form symmetry, and in order to capture the dynamics of the full system we need to consider terms that break this 
symmetry weakly. Similarly, as we had to use an element of $S^{1}$ in order to get the reflection symmetry $(x, y) \mapsto(-x, y)$ of the restricted system, this too is only a normal form symmetry. Thus the restricted system does not have a true $\mathbb{D}_{4}$ symmetry, and we must consider breaking it weakly. The invariant subspaces in table 4 with true $\mathbb{D}_{4}$ symmetry are 2-9.

We can reduce the case of subspace 1 to a previously studied system. Note first that the symmetries

$$
(x, y) \mapsto(y, x) \quad \text { and } \quad(x, y) \mapsto(-x,-y)
$$

are true symmetries, with the latter corresponding to two applications of the translation (6). Defining

$$
u=x+y \quad \text { and } \quad v=x-y,
$$

the $(u, v)$ system has $\mathbb{D}_{4} \times S^{1}$ symmetry, but now the normal form symmetries that are weakly broken are the $S^{1}$ and the interchange $(u, v) \mapsto(v, u)$. The problem of the $\mathbb{D}_{4}$-symmetric Hopf bifurcation with weakly broken interchange symmetry has been studied in [26], where they find an incredibly rich variety of dynamics, including bursting phenomena (as a result of a heteroclinic connection to states with infinite amplitude), even when the time symmetry is not broken. With the time symmetry broken as well we expect even richer dynamics [27].

In [26] the bursting is seen as a consequence of a forced breaking of the interchange symmetry, e.g. as a result of distant boundaries breaking a translation symmetry, or of boundaries which are not quite square. In contrast, on the FCC lattice the system still has the exact spatial symmetry of the cubic lattice, and the breaking is a result of the finite ratio $\lambda / \omega$ of timescales, as is explained (for the $S^{1}$ symmetry breaking) in [18].

\section{The Hopf bifurcation on the BCC lattice}

We turn now to the Hopf bifurcation on the BCC lattice. We have 12 critical wavevectors $\pm \boldsymbol{k}_{j}$ pointing to the midpoints of the edges of a cube, with

$$
\begin{aligned}
& k_{1}=\frac{k_{\mathrm{c}}}{\sqrt{2}}(1,1,0), \quad \boldsymbol{k}_{2}=\frac{k_{\mathrm{c}}}{\sqrt{2}}(0,1,1), \quad \boldsymbol{k}_{3}=\frac{k_{\mathrm{c}}}{\sqrt{2}}(1,0,1), \\
& k_{4}=\frac{k_{\mathrm{c}}}{\sqrt{2}}(1,-1,0), \quad \boldsymbol{k}_{5}=\frac{k_{\mathrm{c}}}{\sqrt{2}}(0,1,-1), \quad \boldsymbol{k}_{6}=\frac{k_{\mathrm{c}}}{\sqrt{2}}(-1,0,1) .
\end{aligned}
$$

A typical scalar takes the form of equation (1). The normal form is, to cubic order,

$$
\begin{aligned}
\dot{z}_{1}=(\lambda+\mathrm{i} \omega) z_{1} & +a\left|z_{1}\right|^{2} z_{1}+b\left|w_{1}\right|^{2} z_{1}+c\left(\left|w_{4}\right|^{2}+\left|z_{4}\right|^{2}\right) z_{1}+d \bar{w}_{1} w_{4} z_{4} \\
& +e\left(\left|w_{6}\right|^{2}+\left|z_{2}\right|^{2}+\left|z_{3}\right|^{2}+\left|z_{5}\right|^{2}\right) z_{1}+f\left(\left|w_{2}\right|^{2}+\left|w_{3}\right|^{2}+\left|w_{5}\right|^{2}+\left|z_{6}\right|^{2}\right) z_{1} \\
& +g\left(w_{2} z_{2}+w_{3} z_{3}+w_{5} z_{5}+w_{6} z_{6}\right) \bar{w}_{1}+h\left(w_{6} z_{3} \bar{z}_{4}+\bar{w}_{4} z_{2} z_{5}\right) \\
& +j\left(\bar{w}_{3} w_{4} w_{6}+\bar{w}_{5} z_{2} z_{4}+\bar{w}_{2} z_{4} z_{5}+w_{4} z_{3} \bar{z}_{6}\right)
\end{aligned}
$$

for $\lambda$ and $\omega$ arbitrary real and $a, \ldots, j$ arbitrary complex coefficients.

We next find the $\mathbb{C}$-axial subgroups. Although the spatial symmetry group $G=T^{3} \rtimes \mathbb{O} \oplus \mathbb{Z}_{2}$ is the same as for the FCC lattice, the representation $\Gamma_{1}$ is now 12-dimensional and acts upon the amplitudes $z=\left(z_{1}, w_{1} ; \ldots ; z_{6}, w_{6}\right)$. While $T^{3}$ and $\mathbb{Z}_{2}$ still act via equations (2) and (3), the octahedral group acts as the permutations of the edges of a cube.

The normal form has the symmetry $\tilde{\Gamma}_{1}=\Gamma_{1} \times S^{1}$. In order to find the $\mathbb{C}$-axial subgroups we enlarge $\tilde{\Gamma}_{1}$ to a larger group $\tilde{\Gamma}_{2}$. We do this by first rearranging the amplitudes. Henceforth we will write the vector of amplitudes as

$$
\boldsymbol{z}=\left(z_{1}, w_{1} ; z_{4}, w_{4}\left|z_{2}, w_{2} ; z_{5}, w_{5}\right| z_{3}, w_{3} ; z_{6}, w_{6}\right) .
$$


We consider these to be three subsystems, each with two orthogonal sets of wavevectors $\boldsymbol{k}_{j}, \boldsymbol{k}_{j+3}, j=1,2,3$. Due to the relative orientation of the wavevectors the group $\mathbb{O}$ permutes the subsystems without splitting them up. That is, for instance, any permutation that carries $z_{1}$ to the second subsystem also carries $w_{1}, z_{4}$ and $w_{4}$ to the second subsystem. Looking only at the elements of $\Gamma_{1}$ that leave, say, the subsystem $\left(z_{1}, w_{1} ; z_{4}, w_{4}\right)$ invariant, we find that the action on that subsystem resembles $T^{2} \rtimes \mathbb{D}_{4}$, the symmetry group of translations and reflections on the square lattice. Note, however, that any $\gamma \in \tilde{\Gamma}_{1}$ that acts nontrivially on one subsystem necessarily acts nontrivially on another.

We choose to enlarge $\tilde{\Gamma}_{1}$ to $\tilde{\Gamma}_{2}$ by adding generators that correspond to translations and reflections of individual subsystems, so that each subsystem has an independent $T^{2} \rtimes \mathbb{D}_{4}$ action. The spatial symmetry is now the wreath product group $\left(T^{2} \rtimes \mathbb{D}_{4}\right) 2 S_{3}$, where $S_{3}$ is the global group of permutations of the three subsystems, and the total symmetry group is $\tilde{\Gamma}_{2}=\left[\left(T^{2} \rtimes \mathbb{D}_{4}\right) 2 S_{3}\right] \times S^{1}$. In order to use the method of $[12,23]$ we must first solve the Hopf problem with the symmetry of the local group, i.e. we must find the $\mathbb{C}$-axial subgroups of $\left(T^{2} \rtimes \mathbb{D}_{4}\right) \times S^{1}$. These are known [25] and are shown in table 5. These solutions, together with an understanding of the subgroups of the global group $S_{3}$ (which is fortunately extremely easy), let us use the method of $[12,23]$ to find all the $\mathbb{C}$-axial solutions of $\tilde{\Gamma}_{2}$. There are 21 such solutions, and we list them in table 6. As with the FCC lattice we can then determine the $\mathbb{C}$-axial solutions for $\tilde{\Gamma}_{1}$ that correspond to each solution for $\tilde{\Gamma}_{2}$. There are 33 of these, also shown in table 6.

The spatiotemporal structures of many of these solutions are easily described in terms of the axial solutions for the steady-state bifurcation problem on the BCC lattice found and described in [10]. Many are simply travelling or standing wave versions of steady-state structures. The standing wave versions of the lamellae, rhombic prism, square prism, hexagonal prism, triangular prism, bcc, bcci, 123, A and B states are solutions 7, 8, 12, 9b, 9c, 14a, 14b, 9a, 13b and 13a, respectively. Solutions 1, 3a, 4 and 5c are travelling versions of the lamellae, 123, square prism and A states, respectively. The travelling rhombic prisms $2 \mathrm{a}$ and $2 \mathrm{~b}$ have exactly the same spatial structure, but travel in different directions. The same is true for the travelling B states $5 \mathrm{a}$ and $5 \mathrm{~b}$.

Several solutions oscillate between different steady-state patterns. Solution $3 \mathrm{~b}$ oscillates between hexagonal and triangular prisms, 19a oscillates between the 123 state and hexagonal prisms, and 19b oscillates between the 123 state and triangular prisms. Solution 5d oscillates between the A and B states. Each of solutions 10 and 17 alternates between lamellae with different orientations; in the former the different lamellae are $60^{\circ}$ apart and in the latter $90^{\circ}$. The two alternating rhombic prism solutions $18 \mathrm{a}$ and $18 \mathrm{~b}$ differ in the same way that the alternating rhombic prisms on the FCC lattice do: they have different relative spatial displacements between their two sets of rhombic prisms. The same is true for the two alternating square prism solutions $15 \mathrm{a}$ and $15 \mathrm{~b}$. There are in addition solutions that cycle among three states. The cycling rhombs (solution 11) are completely analogous to the cycling rhombs on the

Table 5. The $\mathbb{C}$-axial solutions to the Hopf bifurcation problem on the square lattice with symmetry $\left(T^{2} \rtimes \mathbb{D}_{4}\right) \times S^{1}$. Here $x \in \mathbb{C}$.

\begin{tabular}{ll}
\hline Name & $\left(z_{1}, w_{1} ; z_{4}, w_{4}\right)$ \\
\hline Travelling rolls & $x(1,0 ; 0,0)$ \\
Travelling squares & $x(1,0 ; 1,0)$ \\
Standing rolls & $x(1,1 ; 0,0)$ \\
Standing squares & $x(1,1 ; 1,1)$ \\
Alternating rolls & $x(1,1 ; \mathrm{i}, \mathrm{i})$ \\
\hline
\end{tabular}


Table 6. The $\mathbb{C}$-axial solutions for the extended group $\tilde{\Gamma}_{2}$ and the original group $\tilde{\Gamma}_{1}$ for the BCC lattice. Here $\xi=\mathrm{e}^{\mathrm{i} \pi / 3}, \chi=\mathrm{e}^{\mathrm{i} \pi / 4}, \rho=\mathrm{e}^{\mathrm{i} \pi / 6}$ and $x \in \mathbb{C}$. The solutions are written as in formula (8). The full names of the $\tilde{\Gamma}_{1}$-solutions are given in table 7 .

\begin{tabular}{|c|c|c|c|c|}
\hline & $\tilde{\Gamma}_{2}$-solution $z$ & $\tilde{\Gamma}_{1}$-solution $z$ & & \\
\hline 1 & $x(1,0 ; 0,0|0,0 ; 0,0| 0,0 ; 0,0)$ & $x(1,0 ; 0,0|0,0 ; 0,0| 0,0 ; 0,0)$ & 1 & $\mathrm{TL}$ \\
\hline \multirow[t]{2}{*}{2} & $x(1,0 ; 0,0|1,0 ; 0,0| 0,0 ; 0,0)$ & $x(1,0 ; 0,0|1,0 ; 0,0| 0,0 ; 0,0)$ & $2 \mathrm{a}$ & TR1 \\
\hline & & $x(1,0 ; 0,0|0,1 ; 0,0| 0,0 ; 0,0)$ & $2 b$ & TR2 \\
\hline \multirow[t]{2}{*}{3} & $x(1,0 ; 0,0|1,0 ; 0,0| 1,0 ; 0,0)$ & $x(1,0 ; 0,0|1,0 ; 0,0| 1,0 ; 0,0)$ & $3 a$ & $\mathrm{~T} 123$ \\
\hline & & $x(0,0 ; 1,0|0,0 ; 1,0| 0,0 ; 1,0)$ & $3 b$ & Hex/Tri \\
\hline 4 & $x(1,0 ; 1,0|0,0 ; 0,0| 0,0 ; 0,0)$ & $x(1,0 ; 1,0|0,0 ; 0,0| 0,0 ; 0,0)$ & 4 & TS \\
\hline \multirow[t]{4}{*}{5} & $x(1,0 ; 1,0|1,0 ; 1,0| 0,0 ; 0,0)$ & $x(1,0 ; 1,0|1,0 ; 0,1| 0,0 ; 0,0)$ & $5 a$ & TB1 \\
\hline & & $x(1,0 ; 1,0|0,0 ; 0,0| 1,0 ; 0,1)$ & $5 b$ & TB2 \\
\hline & & $x(1,0 ;-1,0|0,0 ; 0,0| 1,0 ; 0,1)$ & $5 c$ & TA \\
\hline & & $x(1,0 ; 1,0|0,0 ; 0,0| 0,1 ; 1,0)$ & $5 d$ & $\mathrm{~A} / \mathrm{B}$ \\
\hline 6 & $x(1,0 ; 1,0|1,0 ; 1,0| 1,0 ; 1,0)$ & & & \\
\hline 7 & $x(1,1 ; 0,0|0,0 ; 0,0| 0,0 ; 0,0)$ & $x(1,1 ; 0,0|0,0 ; 0,0| 0,0 ; 0,0)$ & 7 & SL \\
\hline 8 & $x(1,1 ; 0,0|1,1 ; 0,0| 0,0 ; 0,0)$ & $x(1,1 ; 0,0|1,1 ; 0,0| 0,0 ; 0,0)$ & 8 & SR \\
\hline \multirow[t]{3}{*}{9} & $x(1,1 ; 0,0|1,1 ; 0,0| 1,1 ; 0,0)$ & $x(1,1 ; 0,0|1,1 ; 0,0| 1,1 ; 0,0)$ & $9 \mathrm{a}$ & S123 \\
\hline & & $x(0,0 ; 1,1|0,0 ; 1,1| 0,0 ; 1,1)$ & $9 b$ & SHex \\
\hline & & $x(0,0 ; 1,-1|0,0 ; 1,-1| 0,0 ; 1,-1)$ & $9 \mathrm{c}$ & STri \\
\hline 10 & $x(1,1 ; 0,0|\mathrm{i}, \mathrm{i} ; 0,0| 0,0 ; 0,0)$ & $x(1,1 ; 0,0|\mathrm{i}, \mathrm{i} ; 0,0| 0,0 ; 0,0)$ & 10 & AL1 \\
\hline 11 & $x\left(1,1 ; 0,0|\xi, \xi ; 0,0| \xi^{2}, \xi^{2} ; 0,0\right)$ & $x\left(1,1 ; 0,0|\xi, \xi ; 0,0| \xi^{2}, \xi^{2} ; 0,0\right)$ & 11 & $\mathrm{CR}$ \\
\hline 12 & $x(1,1 ; 1,1|0,0 ; 0,0| 0,0 ; 0,0)$ & $(1,1 ; 1,1|0,0 ; 0,0| 0,0 ; 0,0)$ & 12 & SS \\
\hline \multirow[t]{2}{*}{13} & $x(1,1 ; 1,1|1,1 ; 1,1| 0,0 ; 0,0)$ & $x(1,1 ; 1,1|1,1 ; 1,1| 0,0 ; 0,0)$ & $13 \mathrm{a}$ & SB \\
\hline & & $x(1,1 ; 1,1|1,1 ;-1,-1| 0,0 ; 0,0)$ & $13 b$ & SA \\
\hline \multirow[t]{2}{*}{14} & $x(1,1 ; 1,1|1,1 ; 1,1| 1,1 ; 1,1)$ & $x(1,1 ; 1,1|1,1 ; 1,1| 1,1 ; 1,1)$ & $14 \mathrm{a}$ & SBCC \\
\hline & & $x(1,-1 ; 1,-1|1,-1 ; 1,-1| 1,-1 ; 1,-1)$ & $14 \mathrm{~b}$ & SBCCI \\
\hline \multirow[t]{2}{*}{15} & $x(1,1 ; 1,1|\mathrm{i}, \mathrm{i} ; \mathrm{i}, \mathrm{i}| 0,0 ; 0,0)$ & $x(1,1 ; 1,1|\mathrm{i}, \mathrm{i} ; \mathrm{i}, \mathrm{i}| 0,0 ; 0,0)$ & $15 \mathrm{a}$ & AS1 \\
\hline & & $x(1,1 ; 1,1|\mathrm{i}, \mathrm{i} ;-\mathrm{i},-\mathrm{i}| 0,0 ; 0,0)$ & $15 b$ & AS2 \\
\hline \multirow[t]{2}{*}{16} & $x\left(1,1 ; 1,1|\xi, \xi ; \xi, \xi| \xi^{2}, \xi^{2} ; \xi^{2}, \xi^{2}\right)$ & $x\left(1,1 ; 1,1|\xi, \xi ; \xi, \xi| \xi^{2}, \xi^{2} ; \xi^{2}, \xi^{2}\right)$ & $16 \mathrm{a}$ & $\mathrm{CB}$ \\
\hline & & $x\left(1,1 ; \mathrm{i},-\mathrm{i}|\xi, \xi ; \mathrm{i} \xi,-\mathrm{i} \xi| \xi^{2}, \xi^{2} ; \mathrm{i} \xi^{2},-\mathrm{i} \xi^{2}\right)$ & $16 \mathrm{~b}$ & $\mathrm{CA}$ \\
\hline 17 & $x(1,1 ; \mathrm{i}, \mathrm{i}|0,0 ; 0,0| 0,0 ; 0,0)$ & $x(1,1 ; \mathrm{i}, \mathrm{i}|0,0 ; 0,0| 0,0 ; 0,0)$ & 17 & AL2 \\
\hline \multirow[t]{2}{*}{18} & $x(1,1 ; \mathrm{i}, \mathrm{i}|1,1 ; \mathrm{i}, \mathrm{i}| 0,0 ; 0,0)$ & $x(1,1 ; \mathrm{i}, \mathrm{i}|1,1 ; \mathrm{i}, \mathrm{i}| 0,0 ; 0,0)$ & $18 \mathrm{a}$ & AR1 \\
\hline & & $x(1,1 ; \mathrm{i}, \mathrm{i}|1,1 ;-\mathrm{i},-\mathrm{i}| 0,0 ; 0,0)$ & $18 \mathrm{~b}$ & AR2 \\
\hline \multirow[t]{2}{*}{19} & $x(1,1 ; \mathrm{i}, \mathrm{i}|1,1 ; \mathrm{i}, \mathrm{i}| 1,1 ; \mathrm{i}, \mathrm{i})$ & $x(1,1 ; \mathrm{i}, \mathrm{i}|1,1 ; \mathrm{i}, \mathrm{i}| 1,1 ; \mathrm{i}, \mathrm{i})$ & $19 \mathrm{a}$ & $123 / \mathrm{Hex}$ \\
\hline & & $x(1,1 ;-1,1|1,1 ;-1,1| 1,1 ;-1,1)$ & $19 b$ & 123/Tri \\
\hline 20 & $x\left(1,1 ; \mathrm{i}, \mathrm{i}\left|\chi, \chi ; \chi^{3}, \chi^{3}\right| 0,0 ; 0,0\right)$ & & & \\
\hline \multirow[t]{2}{*}{21} & $x\left(1,1 ; \mathrm{i}, \mathrm{i}|\rho, \rho ; \mathrm{i} \rho, \mathrm{i} \rho| \rho^{2}, \rho^{2}, \mathrm{i} \rho^{2}, \mathrm{i} \rho^{2}\right)$ & $x\left(1,1 ; \mathrm{i}, \mathrm{i}|\rho, \rho ;-\mathrm{i} \rho,-\mathrm{i} \rho| \rho^{2}, \rho^{2} ; \mathrm{i} \rho^{2}, \mathrm{i} \rho^{2}\right)$ & $21 \mathrm{a}$ & \\
\hline & & $x\left(1,1 ; 1,-1|\rho, \rho ;-\rho, \rho| \rho^{2}, \rho^{2} ; \rho^{2},-\rho^{2}\right)$ & $21 b$ & \\
\hline
\end{tabular}

FCC lattice, while solution 16a (16b) cycles among B states (A states) with three different orientations. The remaining solutions $21 \mathrm{a}$ and $21 \mathrm{~b}$ are more complicated, and we do not give them names here.

Notice that there is no travelling bcc or bcci solution, nor is there one that alternates between the two, although we might have expected them by analogy with the FCC lattice. However, on the FCC lattice a travelling solution composed of waves travelling in the $\boldsymbol{k}_{1}, \boldsymbol{k}_{2}$, $-\boldsymbol{k}_{3}$ and $-\boldsymbol{k}_{4}$ directions is possible because there exists a single vector (parallel to the $x$-axis) which has the same scalar product with each, and thus points in the direction of travel. This is not the case for the BCC lattice.

The corresponding 33 solution branches are given in table 7 , along with the number of zero eigenvalues guaranteed by equivariance. The stability criteria are determined by the 
Table 7. Solution branches for the $\mathbb{C}$-axial solutions on the BCC lattice in terms of the coefficients of the normal form (7). The subscript $r$ denotes the real part. The column labelled \# is the number of group-theoretically guaranteed zero eigenvalues, and is equal to $4-\operatorname{dim}(\Sigma)$.

\begin{tabular}{|c|c|c|c|}
\hline Branch & Name & $\#$ & Branching equation \\
\hline 1 & Travelling lamellae & 1 & $\lambda=-a_{\mathrm{r}}|x|^{2}$ \\
\hline $2 \mathrm{a}$ & Travelling rhombs 1 & 2 & $\lambda=-\left(a_{\mathrm{r}}+e_{\mathrm{r}}\right)|x|^{2}$ \\
\hline $2 b$ & Travelling rhombs 2 & 2 & $\lambda=-\left(a_{\mathrm{r}}+f_{\mathrm{r}}\right)|x|^{2}$ \\
\hline $3 a$ & Travelling 123 & 3 & $\lambda=-\left(a_{\mathrm{r}}+2 e_{\mathrm{r}}\right)|x|^{2}$ \\
\hline $3 b$ & Hex/tri & 3 & $\lambda=-\left(a_{\mathrm{r}}+2 f_{\mathrm{r}}\right)|x|^{2}$ \\
\hline 4 & Travelling squares & 2 & $\lambda=-\left(a_{\mathrm{r}}+c_{\mathrm{r}}\right)|x|^{2}$ \\
\hline $5 \mathrm{a}$ & Travelling B 1 & 3 & $\lambda=-\left(a_{\mathrm{r}}+c_{\mathrm{r}}+e_{\mathrm{r}}+f_{\mathrm{r}}+j_{\mathrm{r}}\right)|x|^{2}$ \\
\hline $5 b$ & Travelling B 2 & 3 & $\lambda=-\left(a_{\mathrm{r}}+c_{\mathrm{r}}+2 e_{\mathrm{r}}+h_{\mathrm{r}}\right)|x|^{2}$ \\
\hline $5 \mathrm{c}$ & Travelling A & 3 & $\lambda=-\left(a_{\mathrm{r}}+c_{\mathrm{r}}+2 e_{\mathrm{r}}-h_{\mathrm{r}}\right)|x|^{2}$ \\
\hline $5 d$ & $\mathrm{~A} / \mathrm{B}$ & 4 & $\lambda=-\left(a_{\mathrm{r}}+c_{\mathrm{r}}+2 f_{\mathrm{r}}\right)|x|^{2}$ \\
\hline 7 & Standing lamellae & 2 & $\lambda=-\left(a_{\mathrm{r}}+b_{\mathrm{r}}\right)|x|^{2}$ \\
\hline 8 & Standing rhombs & 3 & $\lambda=-\left(a_{\mathrm{r}}+b_{\mathrm{r}}+e_{\mathrm{r}}+f_{\mathrm{r}}+g_{\mathrm{r}}\right)|x|^{2}$ \\
\hline $9 \mathrm{a}$ & Standing 123 & 4 & $\lambda=-\left(a_{\mathrm{r}}+b_{\mathrm{r}}+2 e_{\mathrm{r}}+2 f_{\mathrm{r}}+2 g_{\mathrm{r}}\right)|x|^{2}$ \\
\hline $9 b$ & Standing hex & 3 & $\lambda=-\left(a_{\mathrm{r}}+b_{\mathrm{r}}+2 e_{\mathrm{r}}+2 f_{\mathrm{r}}+2 g_{\mathrm{r}}\right)|x|^{2}$ \\
\hline $9 \mathrm{c}$ & Standing tri & 3 & $\lambda=-\left(a_{\mathrm{r}}+b_{\mathrm{r}}+2 e_{\mathrm{r}}+2 f_{\mathrm{r}}+2 g_{\mathrm{r}}\right)|x|^{2}$ \\
\hline 10 & Alternating lamellae 1 & 3 & $\lambda=-\left(a_{\mathrm{r}}+b_{\mathrm{r}}+e_{\mathrm{r}}+f_{\mathrm{r}}-g_{\mathrm{r}}\right)|x|^{2}$ \\
\hline 11 & Cycling rhombs & 4 & $\lambda=-\left(a_{\mathrm{r}}+b_{\mathrm{r}}+2 e_{\mathrm{r}}+2 f_{\mathrm{r}}-g_{\mathrm{r}}\right)|x|^{2}$ \\
\hline 12 & Standing squares & 3 & $\lambda=-\left(a_{\mathrm{r}}+b_{\mathrm{r}}+2 c_{\mathrm{r}}+d_{\mathrm{r}}\right)|x|^{2}$ \\
\hline $13 \mathrm{a}$ & Standing B & 4 & $\lambda=-\left(a_{\mathrm{r}}+b_{\mathrm{r}}+2 c_{\mathrm{r}}+d_{\mathrm{r}}+2 e_{\mathrm{r}}+2 f_{\mathrm{r}}+2 g_{\mathrm{r}}+h_{\mathrm{r}}+2 j_{\mathrm{r}}\right)|x|^{2}$ \\
\hline $13 b$ & Standing A & 4 & $\lambda=-\left(a_{\mathrm{r}}+b_{\mathrm{r}}+2 c_{\mathrm{r}}+d_{\mathrm{r}}+2 e_{\mathrm{r}}+2 f_{\mathrm{r}}+2 g_{\mathrm{r}}-h_{\mathrm{r}}-2 j_{\mathrm{r}}\right)|x|^{2}$ \\
\hline $14 \mathrm{a}$ & Standing bcc & 4 & $\lambda=-\left(a_{\mathrm{r}}+b_{\mathrm{r}}+2 c_{\mathrm{r}}+d_{\mathrm{r}}+4 e_{\mathrm{r}}+4 f_{\mathrm{r}}+4 g_{\mathrm{r}}+2 h_{\mathrm{r}}+4 j_{\mathrm{r}}\right)|x|^{2}$ \\
\hline $14 \mathrm{~b}$ & Standing bccI & 4 & $\lambda=-\left(a_{\mathrm{r}}+b_{\mathrm{r}}+2 c_{\mathrm{r}}+d_{\mathrm{r}}+4 e_{\mathrm{r}}+4 f_{\mathrm{r}}+4 g_{\mathrm{r}}-2 h_{\mathrm{r}}-4 j_{\mathrm{r}}\right)|x|^{2}$ \\
\hline $15 \mathrm{a}$ & Alternating squares 1 & 4 & $\lambda=-\left(a_{\mathrm{r}}+b_{\mathrm{r}}+2 c_{\mathrm{r}}+d_{\mathrm{r}}+2 e_{\mathrm{r}}+2 f_{\mathrm{r}}-2 g_{\mathrm{r}}-h_{\mathrm{r}}+2 j_{\mathrm{r}}\right)|x|^{2}$ \\
\hline $15 b$ & Alternating squares 2 & 4 & $\lambda=-\left(a_{\mathrm{r}}+b_{\mathrm{r}}+2 c_{\mathrm{r}}+d_{\mathrm{r}}+2 e_{\mathrm{r}}+2 f_{\mathrm{r}}-2 g_{\mathrm{r}}+h_{\mathrm{r}}-2 j_{\mathrm{r}}\right)|x|^{2}$ \\
\hline $16 \mathrm{a}$ & Cycling B & 4 & $\lambda=-\left(a_{\mathrm{r}}+b_{\mathrm{r}}+2 c_{\mathrm{r}}+d_{\mathrm{r}}+4 e_{\mathrm{r}}+4 f_{\mathrm{r}}-2 g_{\mathrm{r}}-h_{\mathrm{r}}+4 j_{\mathrm{r}}\right)|x|^{2}$ \\
\hline $16 \mathrm{~b}$ & Cycling A & 4 & $\lambda=-\left(a_{\mathrm{r}}+b_{\mathrm{r}}+2 c_{\mathrm{r}}+d_{\mathrm{r}}+4 e_{\mathrm{r}}+4 f_{\mathrm{r}}-2 g_{\mathrm{r}}+h_{\mathrm{r}}-4 j_{\mathrm{r}}\right)|x|^{2}$ \\
\hline 17 & Alternating lamellae 2 & 3 & $\lambda=-\left(a_{\mathrm{r}}+b_{\mathrm{r}}+2 c_{\mathrm{r}}-d_{\mathrm{r}}\right)|x|^{2}$ \\
\hline $18 \mathrm{a}$ & Alternating rhombs 1 & 4 & $\lambda=-\left(a_{\mathrm{r}}+b_{\mathrm{r}}+2 c_{\mathrm{r}}-d_{\mathrm{r}}+2 e_{\mathrm{r}}+2 f_{\mathrm{r}}+h_{\mathrm{r}}\right)|x|^{2}$ \\
\hline $18 \mathrm{~b}$ & Alternating rhombs 2 & 4 & $\lambda=-\left(a_{\mathrm{r}}+b_{\mathrm{r}}+2 c_{\mathrm{r}}-d_{\mathrm{r}}+2 e_{\mathrm{r}}+2 f_{\mathrm{r}}-h_{\mathrm{r}}\right)|x|^{2}$ \\
\hline $19 \mathrm{a}$ & 123/hex & 4 & $\lambda=-\left(a_{\mathrm{r}}+b_{\mathrm{r}}+2 c_{\mathrm{r}}-d_{\mathrm{r}}+4 e_{\mathrm{r}}+4 f_{\mathrm{r}}+2 h_{\mathrm{r}}\right)|x|^{2}$ \\
\hline $19 \mathrm{~b}$ & $123 /$ tri & 4 & $\lambda=-\left(a_{\mathrm{r}}+b_{\mathrm{r}}+2 c_{\mathrm{r}}-d_{\mathrm{r}}+4 e_{\mathrm{r}}+4 f_{\mathrm{r}}-2 h_{\mathrm{r}}\right)|x|^{2}$ \\
\hline 21a & & 4 & $\lambda=-\left(a_{\mathrm{r}}+b_{\mathrm{r}}+2 c_{\mathrm{r}}-d_{\mathrm{r}}+4 e_{\mathrm{r}}+4 f_{\mathrm{r}}-h_{\mathrm{r}}\right)|x|^{2}$ \\
\hline $21 b$ & & 4 & $\lambda=-\left(a_{\mathrm{r}}+b_{\mathrm{r}}+2 c_{\mathrm{r}}-d_{\mathrm{r}}+4 e_{\mathrm{r}}+4 f_{\mathrm{r}}+h_{\mathrm{r}}\right)|x|^{2}$ \\
\hline
\end{tabular}

eigenvalues of $\mathrm{d} \boldsymbol{F}$ for each solution, and are given in table 8. (Solutions 21a and 21b proved particularly vexing, and we were unable to determine their stability properties.) Each solution has only as many zero eigenvalues as equivariance requires, except for the standing hexagonal and triangular prisms, which have four each. Presumably one eigenvalue for each depends only upon higher order terms in the normal form, so that their stabilities are not determined by the cubic order truncation we study in this paper.

\section{Discussion}

We have used extension of the symmetry group to find the $\mathbb{C}$-axial solutions to the equivariant Hopf bifurcation problem posed on the FCC and BCC lattices. We have also determined the stability properties of these solutions, and found a number of primary 
Table 8. Stability criteria for the $\mathbb{C}$-axial solutions on the BCC lattice. The subscript $r$ (i) denotes the real (imaginary) part. The solution is orbitally asymptotically stable if each of the terms shown is negative. (Note: $9 \mathrm{~b}$ and $9 \mathrm{c}$ have eigenvalues that are not determined at cubic order in the normal form.)

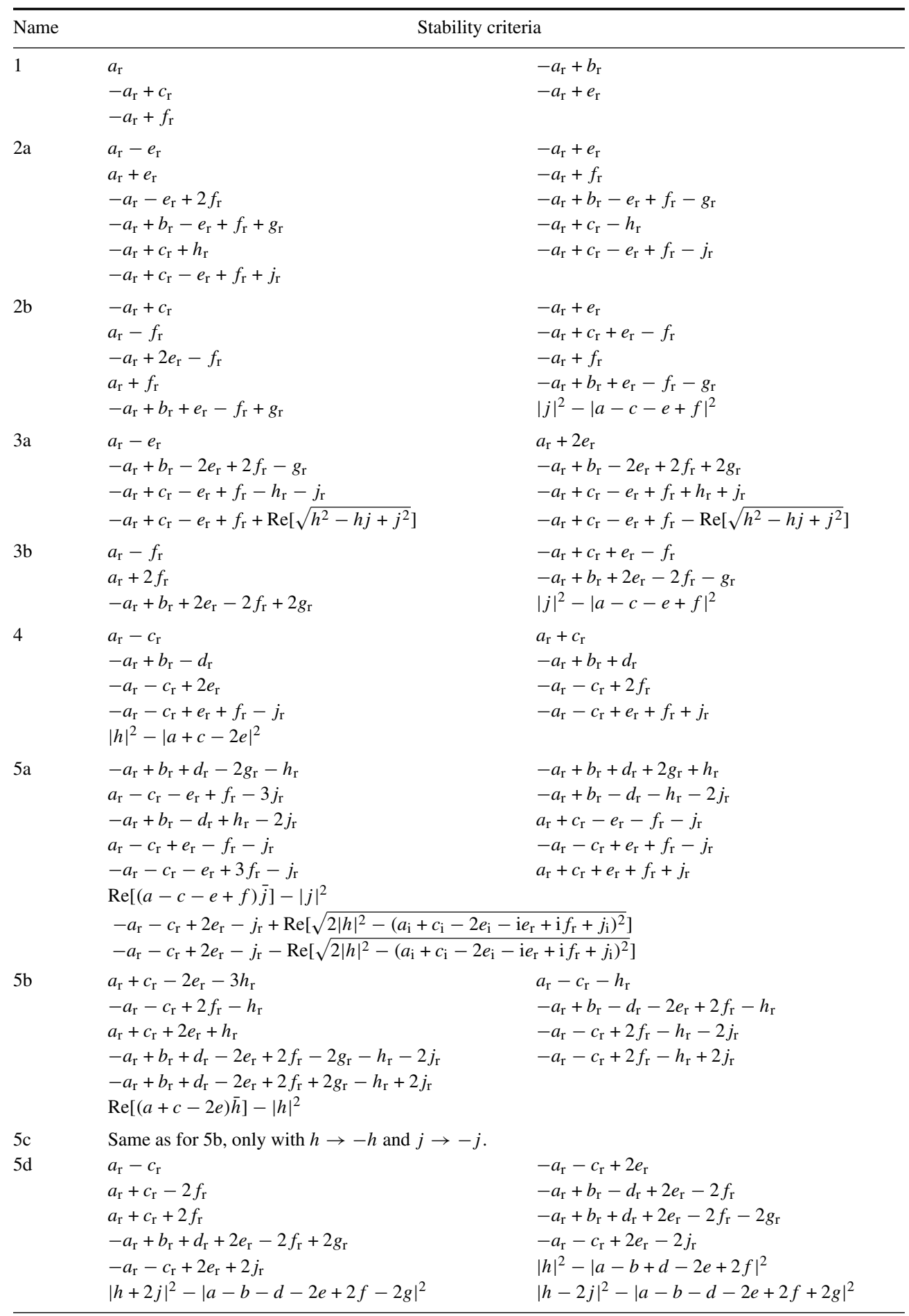


Table 8. (Continued)

\begin{tabular}{|c|c|c|}
\hline \multirow{2}{*}{$\begin{array}{l}\text { Name } \\
7\end{array}$} & \multicolumn{2}{|c|}{ Stability criteria } \\
\hline & $\begin{array}{l}a_{\mathrm{r}}-b_{\mathrm{r}} \\
-a_{\mathrm{r}}-b_{\mathrm{r}}+2 c_{\mathrm{r}} \\
|d|^{2}-|a+b-2 c|^{2}\end{array}$ & $\begin{array}{l}a_{\mathrm{r}}+b_{\mathrm{r}} \\
-a_{\mathrm{r}}-b_{\mathrm{r}}+e_{\mathrm{r}}+f_{\mathrm{r}} \\
|g|^{2}-|a+b-e-f|^{2}\end{array}$ \\
\hline 8 & $\begin{array}{l}a_{\mathrm{r}}+b_{\mathrm{r}}-e_{\mathrm{r}}-f_{\mathrm{r}}-3 g_{\mathrm{r}} \\
a_{\mathrm{r}}-b_{\mathrm{r}}-e_{\mathrm{r}}+f_{\mathrm{r}}-g_{\mathrm{r}} \\
a_{\mathrm{r}}+b_{\mathrm{r}}+e_{\mathrm{r}}+f_{\mathrm{r}}+g_{\mathrm{r}} \\
-a_{\mathrm{r}}-b_{\mathrm{r}}+2 c_{\mathrm{r}}-g_{\mathrm{r}}+h_{\mathrm{r}}+j_{\mathrm{r}} \\
|d+g+j|^{2}-|a+b-2 c+g-h-j|^{2} \\
4|g|^{2}-|a+b-e-f+g|^{2}\end{array}$ & $\begin{array}{l}a_{\mathrm{r}}-b_{\mathrm{r}}+e_{\mathrm{r}}-f_{\mathrm{r}}-g_{\mathrm{r}} \\
-a_{\mathrm{r}}-b_{\mathrm{r}}+e_{\mathrm{r}}+f_{\mathrm{r}}-g_{\mathrm{r}} \\
-a_{\mathrm{r}}-b_{\mathrm{r}}+2 c_{\mathrm{r}}-g_{\mathrm{r}}-h_{\mathrm{r}}-j_{\mathrm{r}} \\
\operatorname{Re}[(a+b-e-f) \bar{g}]-|g|^{2} \\
|d+g-j|^{2}-|a+b-2 c+g+h+j|^{2}\end{array}$ \\
\hline $9 \mathrm{a}$ & $\begin{array}{l}a_{\mathrm{r}}+b_{\mathrm{r}}-e_{\mathrm{r}}-f_{\mathrm{r}}-4 g_{\mathrm{r}} \\
a_{\mathrm{r}}-b_{\mathrm{r}}-e_{\mathrm{r}}+f_{\mathrm{r}}-2 g_{\mathrm{r}} \\
-a_{\mathrm{r}}-b_{\mathrm{r}}+2 c_{\mathrm{r}}-2 g_{\mathrm{r}}-2 h_{\mathrm{r}}-2 j_{\mathrm{r}} \\
-a_{\mathrm{r}}-b_{\mathrm{r}}+2 c_{\mathrm{r}}-2 g_{\mathrm{r}}+h_{\mathrm{r}}+j_{\mathrm{r}} \\
|d+2 g-j|^{2}-|a+b-2 c+2 g+h+j|^{2} \\
|d+2 g+j|^{2}-|a+b-2 c+2 g-h-j|^{2} \\
|d+2 g+2 j|^{2}-|a+b-2 c+2 g-2 h-2 j|^{2} \\
|d+2 g-2 j|^{2}-|a+b-2 c+2 g+2 h+2 j|^{2} \\
\operatorname{Re}[(a+b-e-f) \bar{g}]-|g|^{2}\end{array}$ & $\begin{array}{l}a_{\mathrm{r}}-b_{\mathrm{r}}+2 e_{\mathrm{r}}-2 f_{\mathrm{r}}-2 g_{\mathrm{r}} \\
a_{\mathrm{r}}+b_{\mathrm{r}}+2 e_{\mathrm{r}}+2 f_{\mathrm{r}}+2 g_{\mathrm{r}} \\
-a_{\mathrm{r}}-b_{\mathrm{r}}+2 c_{\mathrm{r}}-2 g_{\mathrm{r}}-h_{\mathrm{r}}-j_{\mathrm{r}} \\
-a_{\mathrm{r}}-b_{\mathrm{r}}+2 c_{\mathrm{r}}-2 g_{\mathrm{r}}+2 h_{\mathrm{r}}+2 j_{\mathrm{r}}\end{array}$ \\
\hline $9 b$ & $\begin{array}{l}a_{\mathrm{r}}+b_{\mathrm{r}}-e_{\mathrm{r}}-f_{\mathrm{r}}-4 g_{\mathrm{r}} \\
a_{\mathrm{r}}-b_{\mathrm{r}}-2 e_{\mathrm{r}}+2 f_{\mathrm{r}}-2 g_{\mathrm{r}} \\
-a_{\mathrm{r}}-b_{\mathrm{r}}+2 c_{\mathrm{r}}-2 g_{\mathrm{r}}-h_{\mathrm{r}}-j_{\mathrm{r}} \\
|d+2 g+2 j|^{2}-|a+b-2 c+2 g-2 h-2 j|^{2} \\
|d+2 g-j|^{2}-|a+b-2 c+2 g+h+j|^{2} \\
\operatorname{Re}[(a+b-e-f) \bar{g}]-|g|^{2}\end{array}$ & $\begin{array}{l}a_{\mathrm{r}}-b_{\mathrm{r}}+e_{\mathrm{r}}-f_{\mathrm{r}}-2 g_{\mathrm{r}} \\
a_{\mathrm{r}}+b_{\mathrm{r}}+2 e_{\mathrm{r}}+2 f_{\mathrm{r}}+2 g_{\mathrm{r}} \\
-a_{\mathrm{r}}-b_{\mathrm{r}}+2 c_{\mathrm{r}}-2 g_{\mathrm{r}}+2 h_{\mathrm{r}}+2 j_{\mathrm{r}}\end{array}$ \\
\hline $9 \mathrm{c}$ & Same as for $9 \mathrm{~b}$, only with $h \rightarrow-h$ and $j \rightarrow-j$ & \\
\hline 10 & $\begin{array}{l}a_{\mathrm{r}}+b_{\mathrm{r}}+e_{\mathrm{r}}+f_{\mathrm{r}}-g_{\mathrm{r}} \\
a_{\mathrm{r}}-b_{\mathrm{r}}-e_{\mathrm{r}}+f_{\mathrm{r}}+g_{\mathrm{r}} \\
a_{\mathrm{r}}+b_{\mathrm{r}}-e_{\mathrm{r}}-f_{\mathrm{r}}+3 g_{\mathrm{r}} \\
-a_{\mathrm{r}}-b_{\mathrm{r}}+2 c_{\mathrm{r}}+g_{\mathrm{r}}-h_{\mathrm{r}}+j_{\mathrm{r}} \\
|d-g+j|^{2}-|a+b-2 c-g+h-j|^{2} \\
|d-g-j|^{2}-|a+b-2 c-g-h+j|^{2}\end{array}$ & $\begin{array}{l}a_{\mathrm{r}}-b_{\mathrm{r}}+e_{\mathrm{r}}-f_{\mathrm{r}}+g_{\mathrm{r}} \\
-a_{\mathrm{r}}-b_{\mathrm{r}}+e_{\mathrm{r}}+f_{\mathrm{r}}+g_{\mathrm{r}} \\
-a_{\mathrm{r}}-b_{\mathrm{r}}+2 c_{\mathrm{r}}+g_{\mathrm{r}}+h_{\mathrm{r}}-j_{\mathrm{r}} \\
\operatorname{Re}[(-a-b+e+f) \bar{g}]-|g|^{2}\end{array}$ \\
\hline 11 & $\begin{array}{l}a_{\mathrm{r}}+b_{\mathrm{r}}+2 e_{\mathrm{r}}+2 f_{\mathrm{r}}-g_{\mathrm{r}} \\
a_{\mathrm{r}}-b_{\mathrm{r}}-e_{\mathrm{r}}+f_{\mathrm{r}}+g_{\mathrm{r}} \\
a_{\mathrm{r}}+b_{\mathrm{r}}-2 c_{\mathrm{r}}-g_{\mathrm{r}}-h_{\mathrm{r}}+2 j_{\mathrm{r}} \\
|d-g+2 j|^{2}-|a+b-2 c-g+h-2 j|^{2} \\
|d-g-2 j|^{2}-|a+b-2 c-g-h+2 j|^{2} \\
a_{\mathrm{r}}+b_{\mathrm{r}}-e_{\mathrm{r}}-f_{\mathrm{r}}+2 g_{\mathrm{r}}+\operatorname{Re}\left[\sqrt{\left(a_{\mathrm{r}}+b_{\mathrm{r}}-e_{\mathrm{r}}-f_{\mathrm{r}}\right.}\right. \\
a_{\mathrm{r}}+b_{\mathrm{r}}-e_{\mathrm{r}}-f_{\mathrm{r}}+2 g_{\mathrm{r}}-\operatorname{Re}\left[\sqrt{\left(a_{\mathrm{r}}+b_{\mathrm{r}}-e_{\mathrm{r}}-f_{\mathrm{r}}\right.}\right. \\
-2 a_{\mathrm{r}}-2 b_{\mathrm{r}}+4 c_{\mathrm{r}}+2 g_{\mathrm{r}}+h_{\mathrm{r}}-2 j_{\mathrm{r}} \\
\quad+\operatorname{Re}\left[\sqrt{4|d-g-j|^{2}-\left(2 a_{\mathrm{i}}+2 b_{\mathrm{i}}-4 c_{\mathrm{i}}-2 g_{\mathrm{i}}\right.}\right. \\
-2 a_{\mathrm{r}}-2 b_{\mathrm{r}}+4 c_{\mathrm{r}}+2 g_{\mathrm{r}}+h_{\mathrm{r}}-2 j_{\mathrm{r}} \\
\quad-\operatorname{Re}\left[\sqrt{4|d-g-j|^{2}-\left(2 a_{\mathrm{i}}+2 b_{\mathrm{i}}-4 c_{\mathrm{i}}-2 g_{\mathrm{i}}\right.}\right. \\
-2 a_{\mathrm{r}}-2 b_{\mathrm{r}}+4 c_{\mathrm{r}}+2 g_{\mathrm{r}}-h_{\mathrm{r}}+2 j_{\mathrm{r}} \\
\quad+\operatorname{Re}\left[\sqrt{4|d-g+j|^{2}-\left(2 a_{\mathrm{i}}+2 b_{\mathrm{i}}-4 c_{\mathrm{i}}-2 g_{\mathrm{i}}\right.}+\right. \\
-2 a_{\mathrm{r}}-2 b_{\mathrm{r}}+4 c_{\mathrm{r}}+2 g_{\mathrm{r}}-h_{\mathrm{r}}+2 j_{\mathrm{r}} \\
\quad-\operatorname{Re}\left[\sqrt{4|d-g+j|^{2}-\left(2 a_{\mathrm{i}}+2 b_{\mathrm{i}}-4 c_{\mathrm{i}}-2 g_{\mathrm{i}}+\right.}\right.\end{array}$ & 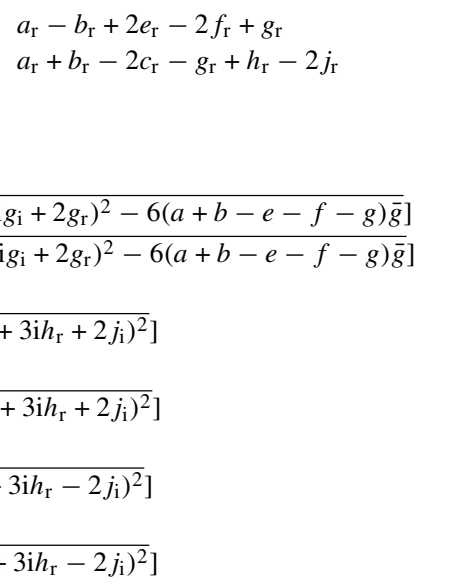 \\
\hline 12 & $\begin{array}{l}a_{\mathrm{r}}+b_{\mathrm{r}}-2 c_{\mathrm{r}}-3 d_{\mathrm{r}} \\
-a_{\mathrm{r}}-b_{\mathrm{r}}-2 c_{\mathrm{r}}-d_{\mathrm{r}}+2 e_{\mathrm{r}}+2 f_{\mathrm{r}}-2 j_{\mathrm{r}} \\
-a_{\mathrm{r}}-b_{\mathrm{r}}-2 c_{\mathrm{r}}-d_{\mathrm{r}}+2 e_{\mathrm{r}}+2 f_{\mathrm{r}}+2 j_{\mathrm{r}}\end{array}$ & $\begin{array}{l}a_{\mathrm{r}}-b_{\mathrm{r}}-d_{\mathrm{r}} \\
a_{\mathrm{r}}+b_{\mathrm{r}}+2 c_{\mathrm{r}}+d_{\mathrm{r}} \\
\operatorname{Re}[(a+b-2 c) \bar{d}]-|d|^{2}\end{array}$ \\
\hline
\end{tabular}


Table 8. (Continued)

\begin{tabular}{|c|c|}
\hline Name & Stability criteria \\
\hline & $\begin{array}{l}|2 g-h|^{2}-|a+b+2 c+d-2 e-2 f+2 j|^{2} \\
|2 g+h|^{2}-|a+b+2 c+d-2 e-2 f-2 j|^{2}\end{array}$ \\
\hline $13 \mathrm{a}$ & $\begin{array}{l}a_{\mathrm{r}}-b_{\mathrm{r}}-d_{\mathrm{r}}-2 g_{\mathrm{r}}-h_{\mathrm{r}} \\
-a_{\mathrm{r}}+b_{\mathrm{r}}+d_{\mathrm{r}}+2 e_{\mathrm{r}}-2 f_{\mathrm{r}}+2 g_{\mathrm{r}}+3 h_{\mathrm{r}}+6 j_{\mathrm{r}} \\
-a_{\mathrm{r}}-b_{\mathrm{r}}+2 c_{\mathrm{r}}+3 d_{\mathrm{r}}+2 g_{\mathrm{r}}+h_{\mathrm{r}}+4 j_{\mathrm{r}} \\
-a_{\mathrm{r}}-b_{\mathrm{r}}-2 c_{\mathrm{r}}-d_{\mathrm{r}}+2 e_{\mathrm{r}}+2 f_{\mathrm{r}}+6 g_{\mathrm{r}}+3 h_{\mathrm{r}}+2 j_{\mathrm{r}} \\
a_{\mathrm{r}}-b_{\mathrm{r}}-d_{\mathrm{r}}+2 e_{\mathrm{r}}-2 f_{\mathrm{r}}-2 g_{\mathrm{r}}+h_{\mathrm{r}}-2 j_{\mathrm{r}} \\
a_{\mathrm{r}}+b_{\mathrm{r}}+2 c_{\mathrm{r}}+d_{\mathrm{r}}-2 e_{\mathrm{r}}-2 f_{\mathrm{r}}+2 g_{\mathrm{r}}+h_{\mathrm{r}}-2 j_{\mathrm{r}} \\
-a_{\mathrm{r}}-b_{\mathrm{r}}-2 c_{\mathrm{r}}-d_{\mathrm{r}}+2 e_{\mathrm{r}}+2 f_{\mathrm{r}}-2 g_{\mathrm{r}}-h_{\mathrm{r}}-2 j_{\mathrm{r}} \\
a_{\mathrm{r}}+b_{\mathrm{r}}+2 c_{\mathrm{r}}+d_{\mathrm{r}}+2 e_{\mathrm{r}}+2 f_{\mathrm{r}}+2 g_{\mathrm{r}}+h_{\mathrm{r}}+2 j_{\mathrm{r}} \\
a_{\mathrm{r}}+b_{\mathrm{r}}+2 c_{\mathrm{r}}+d_{\mathrm{r}}-2 e_{\mathrm{r}}-2 f_{\mathrm{r}}+2 g_{\mathrm{r}}+h_{\mathrm{r}}+6 j_{\mathrm{r}} \\
\operatorname{Re}[(a-b-d-2 e+2 f-2 g)(\bar{h}+2 \bar{j})]-|h+2 j|^{2} \\
4|2 g+h|^{2}-|a+b+2 c+d-2 e-2 f+2 g+h-2 j|^{2} \\
4|2 g-h|^{2}-|a+b+2 c+d-2 e-2 f+2 g+h+6 j|^{2} \\
16|g|^{2}-|a+b+2 c+d-2 e-2 f+2 g+h+2 j|^{2} \\
\operatorname{Re}[(a+b-2 c-d-h-2 j)(\bar{d}+\bar{g}+\bar{j})] \\
\operatorname{Re}[(a+b+2 c+d-2 e-2 f-2 g-h-2 j)(2 \bar{g}+\bar{h})]\end{array}$ \\
\hline $13 \mathrm{~b}$ & Same as for $13 \mathrm{a}$, only with $h \rightarrow-h$ and $j \rightarrow-j$ \\
\hline $14 \mathrm{a}$ & $\begin{array}{l}a_{\mathrm{r}}+b_{\mathrm{r}}-2 c_{\mathrm{r}}-3 d_{\mathrm{r}}-4 g_{\mathrm{r}}-2 h_{\mathrm{r}}-8 j_{\mathrm{r}} \\
a_{\mathrm{r}}-b_{\mathrm{r}}-d_{\mathrm{r}}+2 e_{\mathrm{r}}-2 f_{\mathrm{r}}-4 g_{\mathrm{r}}-2 j_{\mathrm{r}} \\
a_{\mathrm{r}}-b_{\mathrm{r}}-d_{\mathrm{r}}-2 e_{\mathrm{r}}+2 f_{\mathrm{r}}-4 g_{\mathrm{r}}-4 h_{\mathrm{r}}-6 j_{\mathrm{r}} \\
a_{\mathrm{r}}+b_{\mathrm{r}}+2 c_{\mathrm{r}}+d_{\mathrm{r}}-2 e_{\mathrm{r}}-2 f_{\mathrm{r}}-8 g_{\mathrm{r}}-4 h_{\mathrm{r}}-2 j_{\mathrm{r}} \\
a_{\mathrm{r}}+b_{\mathrm{r}}+2 c_{\mathrm{r}}+d_{\mathrm{r}}+4 e_{\mathrm{r}}+4 f_{\mathrm{r}}+4 g_{\mathrm{r}}+2 h_{\mathrm{r}}+4 j_{\mathrm{r}} \\
\operatorname{Re}[(a+b+2 c+d-2 e-2 f-2 j)(2 \bar{g}+\bar{h})]-|2 g+h|^{2} \\
\operatorname{Re}[(a+b-2 c-2 h)(\bar{d}+2 \bar{g}+2 \bar{j})]-2 \operatorname{Re}[(d+4 j) \bar{g}]-|d+3 j|^{2}+|j|^{2} \\
\operatorname{Re}[(a-b-d-2 e+2 f-4 g)(\bar{h}+2 \bar{j})]-|h+3 j|^{2}-|h|^{2}+5|j|^{2}\end{array}$ \\
\hline $14 \mathrm{~b}$ & Same as for $14 \mathrm{a}$, only with $h \rightarrow-h$ and $j \rightarrow-j$ \\
\hline $15 \mathrm{a}$ & $\begin{array}{l}a_{\mathrm{r}}-b_{\mathrm{r}}-d_{\mathrm{r}}+2 g_{\mathrm{r}}+h_{\mathrm{r}} \\
-a_{\mathrm{r}}-b_{\mathrm{r}}-2 c_{\mathrm{r}}-d_{\mathrm{r}}+2 e_{\mathrm{r}}+2 f_{\mathrm{r}}+2 g_{\mathrm{r}}+h_{\mathrm{r}}-6 j_{\mathrm{r}} \\
a_{\mathrm{r}}-b_{\mathrm{r}}-d_{\mathrm{r}}-2 e_{\mathrm{r}}+2 f_{\mathrm{r}}+2 g_{\mathrm{r}}+3 h_{\mathrm{r}}-6 j_{\mathrm{r}} \\
a_{\mathrm{r}}+b_{\mathrm{r}}-2 c_{\mathrm{r}}-3 d_{\mathrm{r}}+2 g_{\mathrm{r}}+h_{\mathrm{r}}-4 j_{\mathrm{r}} \\
a_{\mathrm{r}}-b_{\mathrm{r}}-d_{\mathrm{r}}+2 e_{\mathrm{r}}-2 f_{\mathrm{r}}+2 g_{\mathrm{r}}-h_{\mathrm{r}}-2 j_{\mathrm{r}} \\
a_{\mathrm{r}}+b_{\mathrm{r}}+2 c_{\mathrm{r}}+d_{\mathrm{r}}-2 e_{\mathrm{r}}-2 f_{\mathrm{r}}+6 g_{\mathrm{r}}+3 h_{\mathrm{r}}-2 j_{\mathrm{r}} \\
-a_{\mathrm{r}}-b_{\mathrm{r}}-2 c_{\mathrm{r}}-d_{\mathrm{r}}+2 e_{\mathrm{r}}+2 f_{\mathrm{r}}+2 g_{\mathrm{r}}+h_{\mathrm{r}}-2 j_{\mathrm{r}} \\
a_{\mathrm{r}}+b_{\mathrm{r}}+2 c_{\mathrm{r}}+d_{\mathrm{r}}+2 e_{\mathrm{r}}+2 f_{\mathrm{r}}-2 g_{\mathrm{r}}-h_{\mathrm{r}}+2 j_{\mathrm{r}} \\
-a_{\mathrm{r}}-b_{\mathrm{r}}-2 c_{\mathrm{r}}-d_{\mathrm{r}}+2 e_{\mathrm{r}}+2 f_{\mathrm{r}}+2 g_{\mathrm{r}}+h_{\mathrm{r}}+2 j_{\mathrm{r}} \\
4|h|^{2}-|a+b+2 c+d-2 e-2 f-2 g-h+2 j|^{2} \\
|a+b-2 c+d-2 g+h|^{2}-|a+b-2 c-3 d+2 g+h-4 j|^{2} \\
\operatorname{Re}[(-a+b+d+2 e-2 f-2 g)(\bar{h}-2 \bar{j})]-|h-2 j|^{2} \\
\operatorname{Re}[(-a-b-2 c-d+2 e+2 f+2 j)(2 \bar{g}+\bar{h})]-|2 g+h|^{2}\end{array}$ \\
\hline $15 b$ & Same as for $15 \mathrm{a}$, only with $h \rightarrow-h$ and $j \rightarrow-j$. \\
\hline \multirow[t]{3}{*}{$16 \mathrm{a}$} & $\begin{array}{l}a_{\mathrm{r}}+b_{\mathrm{r}}-2 c_{\mathrm{r}}-3 d_{\mathrm{r}}+2 g_{\mathrm{r}}+h_{\mathrm{r}}-8 j_{\mathrm{r}} \\
a_{\mathrm{r}}+b_{\mathrm{r}}+2 c_{\mathrm{r}}+d_{\mathrm{r}}+4 e_{\mathrm{r}}+4 f_{\mathrm{r}}-2 g_{\mathrm{r}}-h_{\mathrm{r}}+4 j_{\mathrm{r}} \\
a_{\mathrm{r}}+b_{\mathrm{r}}+2 c_{\mathrm{r}}+d_{\mathrm{r}}-2 e_{\mathrm{r}}-2 f_{\mathrm{r}}+4 g_{\mathrm{r}}+2 h_{\mathrm{r}}-2 j_{\mathrm{r}}\end{array}$ \\
\hline & $\begin{array}{l}\quad+\operatorname{Re}\left[\begin{array}{c}\left(a_{\mathrm{r}}+b_{\mathrm{r}}+2 c_{\mathrm{r}}+d_{\mathrm{r}}-2 e_{\mathrm{r}}-2 f_{\mathrm{r}}+4 g_{\mathrm{r}}+6 \mathrm{i} g_{\mathrm{i}}+2 h_{\mathrm{r}}+3 \mathrm{i} h_{\mathrm{i}}-2 j_{\mathrm{r}}\right)^{2} \\
-6(2 g+h)(\bar{a}+\bar{b}+2 \bar{c}+\bar{d}-2 \bar{e}-2 \bar{f}-2 \bar{g}-\bar{h}-2 \bar{j})\end{array}\right. \\
a_{\mathrm{r}}+b_{\mathrm{r}}+2 c_{\mathrm{r}}+d_{\mathrm{r}}-2 e_{\mathrm{r}}-2 f_{\mathrm{r}}+4 g_{\mathrm{r}}+2 h_{\mathrm{r}}-2 j_{\mathrm{r}}\end{array}$ \\
\hline & $-\operatorname{Re}\left[\begin{array}{c}\sqrt{\left(a_{\mathrm{r}}+b_{\mathrm{r}}+2 c_{\mathrm{r}}+d_{\mathrm{r}}-2 e_{\mathrm{r}}-2 f_{\mathrm{r}}+4 g_{\mathrm{r}}+6 \mathrm{i} g_{\mathrm{i}}+2 h_{\mathrm{r}}+3 \mathrm{i} h_{\mathrm{i}}-2 j_{\mathrm{r}}\right)^{2}} \\
-6(2 g+h)(\bar{a}+\bar{b}+2 \bar{c}+\bar{d}-2 \bar{e}-2 \bar{f}-2 \bar{g}-\bar{h}-2 \bar{j})\end{array}\right.$ \\
\hline
\end{tabular}


Table 8. (Continued)

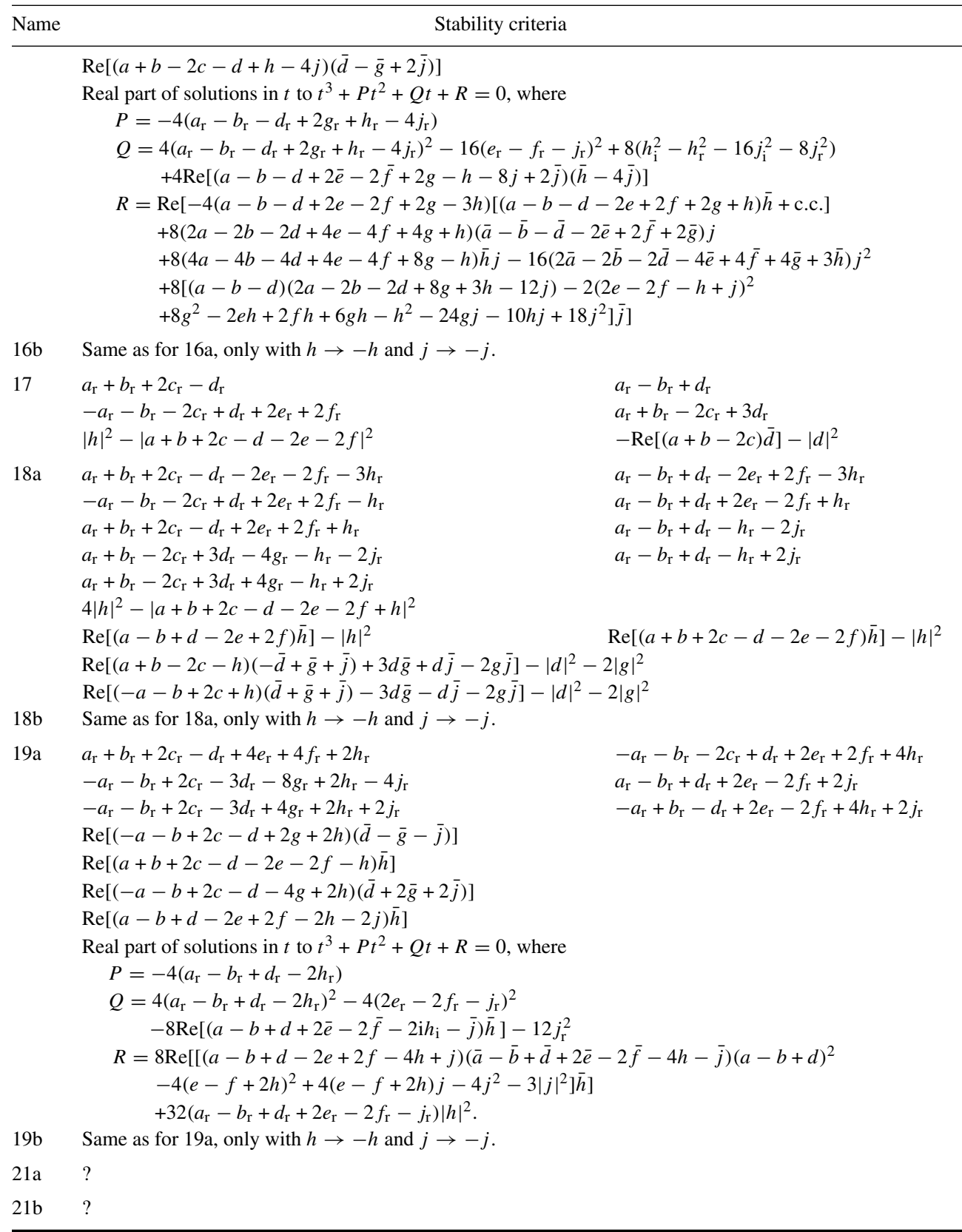

subaxial solutions, with indications of the possibility of quasiperiodic, chaotic and bursting behaviour.

Extension of the symmetry group has been used before to analyse systems, in two contexts. In the first the physical system has an approximate symmetry that is weakly broken. For instance, in [2] Bénard convection is studied as a steady-state bifurcation on the hexagonal lattice. The presence of quadratic terms guarantees that no solutions can be stable at onset [28], 
but can only acquire stability through secondary bifurcations. By assuming a Boussinesq fluid and identical top and bottom boundary conditions an additional $\mathbb{Z}_{2}$ symmetry is obtained, corresponding to reflection through the horizontal midplane. After the solutions are found the $\mathbb{Z}_{2}$ symmetry can be broken weakly, and the resulting unfolding about the Boussinesq case reveals the nonBoussinesq solutions.

The second context where symmetry extension has been commonly used is the case of hidden symmetries [29]. For example, a reaction-diffusion experiment taking place in a rectangular box with Neumann boundary conditions can exhibit behaviour not predicted on the basis of the spatial symmetry of the box alone. The domain can be extended by reflection to a larger box with periodic boundary conditions, and the symmetry group of the system thereby extended to one with continuous translations. The solutions to this problem are then found, and the ones consistent with the original domain kept. Hidden symmetries are also employed when an invariant subspace has a larger symmetry than the whole system, as in [30,31].

In both of these contexts the group extension is motivated by some physical property of the system. In the former the convection system almost has an additional symmetry, which is weakly broken to produce secondary bifurcations. In the latter the boundary conditions or invariant subspaces motivate the extension, which restricts the normal form. In our case we use group extension to find the $\mathbb{C}$-axial solutions. The symmetries we add are not motivated by any physical consideration whatsoever, but are chosen merely for mathematical convenience. Because our added symmetries are arbitrary and need have nothing to do with the physical system, we are not constrained to use weak symmetry breaking to find the solutions to the original problem.

What we have yet to explain is how the appropriate group extensions were determined in the first place. It may seem serendipitous that the Hopf bifurcations on the FCC and BCC lattices admit of easy extensions, but as we shall see we expect many equivariant problems to be solvable in this way.

We wish to extend a given representation $\Gamma_{1}$ of a spatial symmetry group to a representation $\Gamma_{2}$ of a larger group. If $\Gamma_{1}$ is an $N$-dimensional orthogonal representation then it is always possible to extend to $\Gamma_{2}=O(N) \supset \Gamma_{1}$. However, $\tilde{\Gamma}_{2}=\Gamma_{2} \times S^{1}$ then has only one $\mathbb{C}$-axial isotropy subgroup, which must correspond to all of the solutions in $\tilde{\Gamma}_{1}$, so the reduction from $\tilde{\Gamma}_{2}$-solutions to $\tilde{\Gamma}_{1}$-solutions is excessively complicated. If possible, we would like our extension to be minimal in some sense. We would also prefer that $\Gamma_{2}=L$ 々 $G$ be the wreath product of the representation $L$ of some local group and the representation $G$ of some global permutation group, simply because we have a method for determining the $\mathbb{C}$-axial solutions in this case.

We impose a partial ordering on wreath products by defining $\left(L_{2} G\right) \preccurlyeq\left(L^{\prime}{ }_{2} G^{\prime}\right)$ if $L \subseteq L^{\prime}$ and $G \subseteq G^{\prime}$. For this partial ordering we have several minimal extensions. In fact, we have one for each consistent partition of the system into subsystems. By a 'consistent partition' we mean that if $z_{i}$ and $z_{j}$ are both in subsystem $m$, then any $g \in G$ that takes $z_{i}$ to an amplitude in subsystem $n$ must also take $z_{j}$ to an amplitude in subsystem $n$. That is, the permutations do not break up the subsystems.

Consider the Hopf bifurcation on the BCC lattice. To determine the consistent partitions we examine the subsystem that contains $z_{1}$. Suppose it also contains $z_{4}$. There is a symmetry that leaves $z_{1}$ fixed, yet sends $z_{4} \rightarrow w_{4}$, so $z_{1}$ and $w_{4}$ are in the same subsystem. Then there is a symmetry that leaves $w_{4}$ fixed but sends $z_{1} \rightarrow w_{1}$. By this technique we find that there are only four consistent partitions, which we list in table 9 . The fourth partition is not an extension at all and the first has 12 continuous symmetries, which renders the reduction from solutions on $\tilde{\Gamma}_{2}$ to those on $\tilde{\Gamma}_{1}$ needlessly complicated. The second and third each have six continuous symmetries, but the third has the less complicated global group. The Hopf bifurcation on the 
local group $T^{2} \rtimes \mathbb{D}_{4}$ has already been solved, and the method of [23] requires knowing all about the nature of the subgroups of the global group, so we chose to use the third option with $G=S_{3}$.

For the FCC lattice the consistent partitions are listed in table 10. As before, the first and last partitions are not useful. The second partition adds only one extra continuous symmetry and is the one we used here. The third one adds three but is still tractable, and must produce the same solutions.

This method can be applied to other complex systems, and can be used to find solutions much more easily than has been done before. Consider, for example, the Hopf bifurcation problem with the higher-dimensional representation of the square lattice symmetry $\Gamma_{1}=T^{2} \rtimes \mathbb{D}_{4}$ studied in $[3,30]$. The eight critical wavevectors are $\pm \boldsymbol{k}_{j}$, with

$$
\boldsymbol{k}_{1}=(m, n), \quad \boldsymbol{k}_{2}=(n, m), \quad \boldsymbol{k}_{3}=(-n, m), \quad \boldsymbol{k}_{4}=(-m, n)
$$

for integers $m>n>0$ with $\operatorname{gcd}(m, n)=1$. There are 10 consistent partitions, which we list in table 11 . The ninth partition $\left(z_{1}, w_{1}, z_{3}, w_{3} \mid z_{2}, w_{2}, z_{4}, w_{4}\right)$ has the very simple global

Table 9. Consistent partitions for the BCC lattice, together with the corresponding extended groups $\Gamma_{2}$ and the number of continuous symmetries of $\Gamma_{2}$. The first partition uses the 12-dimensional representation of $\mathbb{O}$ on vectors, while the second uses the six-dimensional representation on directors.

\begin{tabular}{llr}
\hline Partition & $\Gamma_{2}$ & $N$ \\
\hline$\left(z_{1}\left|z_{2}\right| z_{3}\left|z_{4}\right| z_{5}\left|z_{6}\right| w_{1}\left|w_{2}\right| w_{3}\left|w_{4}\right| w_{5} \mid w_{6}\right)$ & $S O(2) \imath\left(\mathbb{O} \oplus \mathbb{Z}_{2}\right)$ & 12 \\
$\left(z_{1}, w_{1}\left|z_{2}, w_{2}\right| z_{3}, w_{3}\left|z_{4}, w_{4}\right| z_{5}, w_{5} \mid z_{6}, w_{6}\right)$ & $O(2) \imath \mathbb{O}$ & 6 \\
$\left(z_{1}, w_{1} ; z_{4}, w_{4}\left|z_{2}, w_{2} ; z_{5}, w_{5}\right| z_{3}, w_{3} ; z_{6}, w_{6}\right)$ & $\left(T^{2} \rtimes \mathbb{D}_{4}\right) 2 S_{3}=\left[O(2) 2 S_{2}\right] 2 S_{3}$ & 6 \\
$\left(z_{1}, w_{1} ; z_{2}, w_{2} ; z_{3}, w_{3} ; z_{4}, w_{4} ; z_{5}, w_{5} ; z_{6}, w_{6}\right)$ & $\left(T^{3} \rtimes \mathbb{O} \oplus Z_{2}\right) \imath \mathbf{1}$ & 3 \\
\hline
\end{tabular}

Table 10. Consistent partitions for the FCC lattice, together with the corresponding extended groups $\Gamma_{2}$ and the number of continuous symmetries of $\Gamma_{2}$.

\begin{tabular}{lll}
\hline Partition & $\Gamma_{2}$ & $N$ \\
\hline$\left(z_{1}\left|z_{2}\right| z_{3}\left|z_{4}\right| w_{1}\left|w_{2}\right| w_{3} \mid w_{4}\right)$ & $S O(2) \imath\left(\mathbb{O} \oplus \mathbb{Z}_{2}\right)$ & 8 \\
$\left(z_{1}, w_{1}\left|z_{2}, w_{2}\right| z_{3}, w_{3} \mid z_{4}, w_{4}\right)$ & $O(2) \imath S_{4}$ & 4 \\
$\left(z_{1}, z_{2}, z_{3}, z_{4} \mid w_{1}, w_{2}, w_{3}, w_{4}\right)$ & $\left(T^{3} \rtimes \mathbb{T}\right) \imath \mathbb{Z}_{2}$ & 6 \\
$\left(z_{1}, w_{1}, z_{2}, w_{2}, z_{3}, w_{3}, z_{4}, w_{4}\right)$ & $\left(T^{3} \rtimes \mathbb{O} \oplus Z_{2}\right) \imath \mathbf{1}$ & 3 \\
\hline
\end{tabular}

Table 11. Consistent partitions for the eight-dimensional representation of the square lattice, together with the corresponding extended groups $\Gamma_{2}$ and the number of continuous symmetries of $\Gamma_{2}$. The first and last partitions use the eight-dimensional representation of $\mathbb{D}_{4}$, while the others use the four-dimensional representation.

\begin{tabular}{lll}
\hline Partition & $\Gamma_{2}$ & $N$ \\
\hline$\left(z_{1}\left|w_{1}\right| z_{2}\left|w_{2}\right| z_{3}\left|w_{3}\right| z_{4} \mid w_{4}\right)$ & $S O(2) \imath \mathbb{D}_{4}$ & 8 \\
$\left(z_{1}, w_{1}\left|z_{2}, w_{2}\right| z_{3}, w_{3} \mid z_{4}, w_{4}\right)$ & $O(2) \imath \mathbb{D}_{4}$ & 4 \\
$\left(z_{1}, z_{2}\left|w_{1}, w_{2}\right| z_{3}, z_{4} \mid w_{3}, w_{4}\right)$ & $\left(T^{2} \rtimes \mathbb{Z}_{2}\right) \imath \mathbb{D}_{4}$ & 8 \\
$\left(z_{1}, z_{4}\left|w_{1}, w_{4}\right| z_{2}, z_{3} \mid w_{2}, w_{3}\right)$ & $\left(T^{2} \rtimes \mathbb{Z}_{2}\right) \imath \mathbb{D}_{4}$ & 8 \\
$\left(z_{1}, w_{2}\left|z_{2}, w_{1}\right| z_{3}, w_{4} \mid z_{4}, w_{3}\right)$ & $\left(T^{2} \rtimes \mathbb{Z}_{2}\right) \imath \mathbb{D}_{4}$ & 8 \\
$\left(z_{1}, w_{4}\left|z_{4}, w_{1}\right| z_{2}, z_{3} \mid w_{2}, w_{3}\right)$ & $\left(T^{2} \rtimes \mathbb{Z}_{2}\right) \imath \mathbb{D}_{4}$ & 8 \\
$\left(z_{1}, w_{1} ; z_{2}, w_{2} \mid z_{3}, w_{3} ; z_{4}, w_{4}\right)$ & $\left(T^{2} \rtimes \mathbb{D}_{2}\right) \imath \mathbb{Z}_{2}$ & 4 \\
$\left(z_{1}, w_{1} ; z_{4}, w_{4} \mid z_{2}, w_{2} ; z_{3}, w_{3}\right)$ & $\left(T^{2} \rtimes \mathbb{D}_{2}\right) \imath \mathbb{Z}_{2}$ & 4 \\
$\left(z_{1}, w_{1} ; z_{3}, w_{3} \mid z_{2}, w_{2} ; z_{4}, w_{4}\right)$ & $\left(T^{2} \rtimes \mathbb{D}_{4}\right) \imath \mathbb{Z}_{2}$ & 4 \\
$\left(z_{1}, w_{1} ; z_{2}, w_{2} ; z_{3}, w_{3} ; z_{4}, w_{4}\right)$ & $\left(T^{2} \rtimes \mathbb{D}_{4}\right) \imath \mathbf{1}$ & 2 \\
\hline
\end{tabular}


Table 12. $\quad$-axial solutions $z=\left(z_{1}, w_{1} ; z_{3}, w_{3} \mid z_{2}, w_{2} ; z_{4}, w_{4}\right)$ for the extended group $\tilde{\Gamma}_{2}=\left(T^{2} \rtimes \mathbb{D}_{4}\right) \geq \mathbb{Z}_{2}$ and the original group $\tilde{\Gamma}_{1}$ for the eight-dimensional representation of the square lattice.

\begin{tabular}{llll}
\hline & $\tilde{\Gamma}_{2}$-solution $z$ & $\tilde{\Gamma}_{1}$-solution $z$ & \\
\hline 1 & $x(1,0 ; 0,0 \mid 0,0 ; 0,0)$ & $x(1,0 ; 0,0 \mid 0,0 ; 0,0)$ & $\mathrm{TR}$ \\
2 & $x(1,0 ; 1,0 \mid 0,0 ; 0,0)$ & $x(1,0 ; 1,0 \mid 0,0 ; 0,0)$ & $\mathrm{TS}$ \\
3 & $x(1,1 ; 0,0 \mid 0,0 ; 0,0)$ & $x(1,1 ; 0,0 \mid 0,0 ; 0,0)$ & $\mathrm{SR}$ \\
4 & $x(1,1 ; 1,1 \mid 0,0 ; 0,0)$ & $x(1,1 ; 1,1 \mid 0,0 ; 0,0)$ & $\mathrm{SS}$ \\
5 & $x(1,1 ; \mathrm{i}, \mathrm{i} \mid 0,0 ; 0,0)$ & $x(1,1 ; \mathrm{i}, \mathrm{i} \mid 0,0 ; 0,0)$ & $\mathrm{AR}$ \\
6 & $x(1,0 ; 0,0 \mid 1,0 ; 0,0)$ & $x(1,0 ; 0,0 \mid 1,0,0,0)$ & $\mathrm{TRh}_{3}$ \\
& & $x(1,0 ; 0,0 \mid 0,1,0,0)$ & $\mathrm{TRh}_{4}$ \\
& & $x(1,0 ; 0,0 \mid 0,0,1,0)$ & $\mathrm{TRh}_{2}$ \\
7 & $x(1,0 ; 1,0 \mid 1,0 ; 1,0)$ & $x(1,0 ; 0,0 \mid 0,0,0,1)$ & $\mathrm{TRh}_{1}$ \\
8 & $x(1,1 ; 0,0 \mid 1,1 ; 0,0)$ & $x(1,1 ; 0,0 \mid 1,1 ; 0,0)$ & $\mathrm{SRec}_{2}$ \\
& & $x(1,1 ; 0,0 \mid 0,0 ; 1,1)$ & $\mathrm{SRec}_{1}$ \\
9 & $x(1,1 ; 1,1 \mid 1,1 ; 1,1)$ & $x(1,1 ; 1,1 \mid 1,1 ; 1,1)$ & $\mathrm{SSS}$ \\
& & $x(1,1 ; 1,1 \mid-1,-1 ;-1,-1)$ & $\mathrm{SAS}$ \\
10 & $x(1,1 ; \mathrm{i}, \mathrm{i} \mid 1,1 ; \mathrm{i}, \mathrm{i})$ & $x(1,1 ; \mathrm{i}, \mathrm{i} \mid \mathrm{i}, \mathrm{i} ; 1,1)$ & $\mathrm{ASS}$ \\
11 & $x(1,1 ; 0,0 \mid \mathrm{i}, \mathrm{i} ; 0,0)$ & $x(1,1 ; 0,0 \mid \mathrm{i}, \mathrm{i} ; 0,0)$ & $\mathrm{ARec}_{2}$ \\
& & $x(1,1 ; 0,0 \mid 0,0 ; \mathrm{i}, \mathrm{i})$ & $\mathrm{ARec}_{1}$ \\
12 & $x(1,1 ; 1,1 \mid \mathrm{i}, \mathrm{i} ; \mathrm{i}, \mathrm{i})$ & & \\
13 & $x(1,1 ; \mathrm{i}, \mathrm{i} \mid \mathrm{i}, \mathrm{i} ;-1,-1)$ & $x(1,1 ; \mathrm{i}, \mathrm{i} \mid-\mathrm{i},-\mathrm{i} ;-1,-1)$ & $\mathrm{AAS}$ \\
\hline
\end{tabular}

group $\mathbb{Z}_{2}$, while its local group $\left(T^{2} \rtimes \mathbb{D}_{4}\right)$ is the symmetry of the square lattice, whose $\mathbb{C}$-axial solutions are in table 5 [25]. Choosing this partition to define our group extension, we find the $13 \mathbb{C}$-axial solutions listed in table 12 . Upon reduction to the smaller subgroup these reveal the $17 \mathbb{C}$-axial solutions already found and named in $[3,30]$.

We expect that this method will find use for other highly symmetric systems, particularly those with high-dimensional representations.

\section{Acknowledgment}

This work was supported in part by a grant from the Horace H Rackham School of Graduate Studies at the University of Michigan at Ann Arbor.

\section{References}

[1] Golubitsky M, Stewart I and Schaeffer D G 1988 Singularities and Groups in Bifurcation Theory vol II (Berlin: Springer)

[2] Golubitsky M, Swift J W and Knobloch E 1984 Symmetries and pattern selection in Rayleigh-Bénard convection Physica D 10 249-76

[3] Dionne B, Golubitsky M, Silber M and Stewart I 1995 Time-periodic spatially periodic planforms in Euclidean equivariant partial differential equations Phil. Trans. R. Soc. A 352 125-68

[4] Turing A M 1952 The chemical basis of morphogenesis Phil. Trans. R. Soc. B 237 37-72

[5] De Wit A, Borckmans P and Dewel G 1997 Twist grain boundaries in three-dimensional lamellar Turing structures Proc. Natl Acad. Sci. USA 94 12765-8

[6] De Wit A, Dewel G, Borckmans P and Walgraef D 1992 Three-dimensional dissipative structures Physica D 61 289-96

[7] Dionne B 1993 Planforms in three dimensions Z. Angew. Math. Phys. 44 673-94

[8] Callahan T K 1998 Pattern formation in three dimensions PhD Thesis University of California, Berkeley

[9] Callahan T K and Knobloch E 1996 Bifurcations on the fcc lattice Phys. Rev. E 53 3559-62 
[10] Callahan T K and Knobloch E 1997 Symmetry-breaking bifurcations on cubic lattices Nonlinearity 10 1179-216

[11] Roberts M, Swift J W and Wagner D H 1985 The Hopf bifurcation on a hexagonal lattice Multiparameter Bifurcation Theory (Contemporary Mathematics vol 56) ed M Golubitsky and J M Guckenheimer (Providence, RI: American Mathematical Society) pp 283-318

[12] Dias A P S and Stewart I 1999 Hopf bifurcation on a simple cubic lattice Dyn. Stab. Syst. 14 3-55

[13] Callahan T K 2000 Hopf bifurcations on the FCC lattice Proc. Int. Conf. on Differential Equations (Berlin, 1999) vol 1, ed Fiedler et al (Singapore: World Scientific) pp 154-6

[14] Callahan T K 2003 Hopf bifurcations on cubic lattices Bifurcations, Symmetry and Patterns (Trends in Mathematics) ed J Buescu, S B S D Castro, A P S Dias and I S Labouriau (Basel: Birkhauser) pp 123-7

[15] Newell A C and Whitehead J A 1969 Finite bandwidth, finite amplitude convection J. Fluid Mech. 38 279-303

[16] Hoyle R B 1993 Long wavelength instabilities of square patterns Physica D 67 198-223

[17] Callahan T K and Knobloch E 2001 Long-wavelength instabilities of three-dimensional patterns Phys. Rev. E 64036214

[18] Swift J W 1988 Hopf bifurcation with the symmetry of the square Nonlinearity $1333-77$

[19] Wiggins S 1990 Introduction to Applied Nonlinear Dynamical Systems and Chaos (New York: Springer)

[20] Springer T A 1977 Invariant Theory (Lecture Notes in Mathematics vol 585) (Berlin: Springer)

[21] Sturmfels B 1993 Algorithms in Invariant Theory (New York: Springer)

[22] Worfolk P A 1994 Zeros of equivariant vector fields: algorithms for an invariant approach J. Symbolic Comput. 17 487-511

[23] Dias A P S 1998 Hopf bifurcation for wreath products Nonlinearity 11 247-64

[24] Dionne B, Golubitsky M and Stewart I 1996 Coupled cells with internal symmetry: I. Wreath products Nonlinearity $9559-74$

[25] Silber M and Knobloch E 1991 Hopf bifurcation on a square lattice Nonlinearity 4 1063-107

[26] Moehlis J and Knobloch E 1998 Forced symmetry breaking as a mechanism for bursting Phys. Rev. Lett. 80 5329-32

[27] Moehlis J 2003 Personal communication

[28] Ihrig E and Golubitsky M 1984 Pattern selection with $O(3)$ symmetry Physica D 12 1-33

[29] Crawford J D, Golubitsky M, Gomes M G M, Knobloch E and Stewart I 1991 Boundary conditions as symmetry constraints Singularity Theory and its Applications II (Lecture Notes in Mathematics vol 1463) ed M Roberts and I Stewart (Berlin: Springer) pp 63-79

[30] Dawes J H P 2001 Hopf bifurcation on a square superlattice Nonlinearity 14 491-511

[31] Dawes J H P, Matthews P C and Rucklidge A M 2003 Reducible actions of $\mathbb{D}_{4} \times T^{2}$ : superlattice patterns and hidden symmetries Nonlinearity 16 615-45 